\title{
Structural adaptation in the density model.
}

\author{
O.V. Lepski * G. Rebelles * \\ Institut de Mathématique de Marseille \\ Aix-Marseille Université \\ 39, rue F. Joliot-Curie \\ 13453 Marseille, France \\ e-mail: oleg.lepski@univ-amu.fr \\ e-mail: rebelles.gilles@neuf.fr
}

\begin{abstract}
:
This paper deals with non-parametric density estimation on $\mathbb{R}^{2}$ from i.i.d observations. It is assumed that after unknown rotation of the coordinate system the coordinates of the observations are independent random variables whose densities belong to a Hölder class with unknown parameters. The minimax and adaptive minimax theories for this structural statistical model are developed.
\end{abstract}

AMS 2000 subject classifications: 62G05, 62G20.

Keywords and phrases: density estimation, adaptive estimation, kernel estimators, pointwise risk, Hölder class..

\section{Introduction}

Let $\xi \in \mathbb{R}^{2}$ be a random vector having the density $g$ w.r.t the Lebesgue measure. We will assume that the coordinates of $\xi$ are independent and let $X \in \mathbb{R}^{2}$ be the random vector obtained from the relation

$$
X=M \xi, \quad M \in \mathfrak{Q},
$$

where $\mathfrak{Q}$ is the set of all rotational $2 \times 2$-matrices.

Let we observe $n \in \mathbb{N}^{*}$ independent copies of $X$ that is $X^{(n)}=\left(X_{1}, \ldots, X_{n}\right)$. We want to estimate the density of $X$ denoted by $f$ at a given point $x \in \mathbb{R}^{2}$ using the observations $X^{(n)}$. By estimator, we mean any $X^{(n)}$-measurable map $\tilde{f}: \mathbb{R}^{n} \rightarrow \mathbb{R}$. The accuracy of an estimator $\hat{f}$ is measured by the pointwise risk

$$
\mathcal{R}_{n}^{(p)}[\tilde{f}, f]:=\left(\mathbb{E}_{f}|\tilde{f}-f(x)|^{p}\right)^{1 / p}, p \in[1, \infty) .
$$

${ }^{*}$ This work has been carried out in the framework of the Labex Archimède (ANR11-LABX-0033) and of the A*MIDEX project (ANR-11-IDEX-0001-02), funded by the "Investissements d'Avenir" French Government program managed by the French National Research Agency (ANR). 
Here $\mathbb{E}_{f}$ denotes the expectation with respect to the probability measure $\mathbb{P}_{f}$ of the observations $X^{(n)}$.

Let $\mathcal{Q} \subseteq \mathfrak{Q}$ be fixed and let $\mathcal{G}(\beta, L)$ denote the following set of functions.

Definition 1. We say that $g: \mathbb{R}^{2} \rightarrow \mathbb{R}$ belongs to $\mathcal{G}(\beta, L)$ if

(i) $g(\cdot, \cdot)=g_{1}(\cdot) g_{2}(\cdot)$ and $g_{1}, g_{2}: \mathbb{R} \rightarrow \mathbb{R}_{+}$are symmetric probability densities;

(ii) $g_{1}, g_{2}$ belong to the Hölder class $\mathbb{H}(\beta, L), \beta>0, L>0$, on $\mathbb{R}$.

For the reader's convenience the formal definition of $\mathbb{H}(\beta, L)$ is postponed to the end of this section. Here we only mention that $\beta$ is referred to the smoothness of the underlying function while $L$ is the Lipschitz constant. For any $\beta>0, L>0$ introduce the following set of probability densities.

$$
\mathcal{F}(\beta, L, \mathcal{Q})=\left\{f: \mathbb{R}^{2} \rightarrow \mathbb{R}_{+}: f(\bullet)=g\left(M^{T} \bullet\right), g \in \mathcal{G}(\beta, L), M \in \mathcal{Q}\right\} .
$$

In the present paper we will study the minimax and minimax adaptive estimation of the density $f$ over the collection of functional classes $\mathcal{F}(\beta, L, \mathcal{Q})$. To illustrate the interesting feature of the problem at hand let us consider the simplest situation. Assume that the set $\mathcal{Q}$ consists a single element $\boldsymbol{Q}$. In this case we can first obtain new observation sequence $\xi_{1}=Q^{T} X_{1}, \ldots, \xi_{n}=$ $\boldsymbol{Q}^{T} X_{n}$. Noting that the density of $\xi_{1}$ is $g_{1} g_{2}$ we estimate next separately $g_{1}$ and $g_{2}$ from the sequence of the first and second coordinates of $\xi_{1}, \ldots, \xi_{n}$ respectively. In particular one can use the kernel estimation method with properly chosen bandwidth. It will lead to the estimators $\widehat{g}_{1}$ and $\widehat{g}_{2}$. Since $g_{1}, g_{2} \in \mathbb{H}(\beta, L)$ the pointwise minimax accuracy (minimax rate of convergence) of each marginal density will be proportional to $n^{-\frac{\beta}{2 \beta+1}}$. Therefore, the minimax pointwise accuracy in estimating of $g$ provided by the estimator $\widehat{g}(x)=\widehat{g}_{1}\left(x_{1}\right) \widehat{g}_{2}\left(x_{2}\right)$ is proportional to $n^{-\frac{\beta}{2 \beta+1}}$ as well. The estimator for $f(x)=g\left(\boldsymbol{Q}^{T} x\right)$ is then given by $\widehat{f}_{\boldsymbol{Q}}(x)=\widehat{g}\left(\boldsymbol{Q}^{T} x\right)$.

All saying above can be summarized as follows.

Theorem 1. Let $\beta>0, L>0$ and $\boldsymbol{Q} \in \mathfrak{Q}$ be fixed. Then, for any $x \in \mathbb{R}^{2}$ there exists an estimator $\widehat{f}_{\boldsymbol{Q}}(x)$ such that $\forall p \geq 1$

$$
\sup _{\mathcal{F}(\beta, L,\{\boldsymbol{Q}\})} \mathcal{R}_{n}^{(p)}\left[\widehat{f}_{\boldsymbol{Q}}(x), f\right] \lesssim n^{-\frac{\beta}{2 \beta+1}} .
$$

Moreover (here and later inf is taken over all possible estimators) $\forall p \geq 1$

$$
\inf _{\widetilde{f}} \sup _{\mathcal{F}(\beta, L,\{\boldsymbol{Q}\})} \mathcal{R}_{n}^{(p)}[\widetilde{f}, f] \gtrsim n^{-\frac{\beta}{2 \beta+1}} .
$$

The proof of this theorem is straightforward. Moreover its first assertion follows from the results obtained in Proposition 1 presented in Section 3.

imsart-generic ver. 2014/10/16 file: rotation-arXiv.tex date: February 26, 2020 
The assertions of Theorem 1 show that the structural assumption $f(\bullet)=$ $g\left(M^{T} \bullet\right)$ leads to the essential improvement of the accuracy of estimation. Indeed, it is easily seen that $\mathcal{F}(\beta, L, \mathfrak{Q}) \subset \mathbb{H}(\vec{\beta}, \vec{L})$, where $\mathbb{H}(\vec{\beta}, \vec{L})$ is the isotropic Hölder class on $\mathbb{R}^{2}$ with $\vec{\beta}=(\beta, \beta)$ and $\vec{L}=\left(L^{2}, L^{2}\right)$. Recall that the minimax pointwise accuracy on this class is given by $n^{-\frac{\beta}{2 \beta+2}}$ which is much larger than the univariate rate $n^{-\frac{\beta}{2 \beta+1}}$ available under the structural assumption discussed above.

The first problem which we address is the following: do the statements of Theorem 1 remain valid if the cardinality of $\mathcal{Q}$ is larger than 1 ? We remark that the matrix $M$ describing the law of observation is unknown in this case. Therefore, we are talking about the adaptation to unknown rotation of coordinate system (structural adaptation). We will see that the answer on aforementioned question depends heavily on the "massiveness" of the set $\mathcal{Q}$. In particular, Theorem 1 is not valid if $\mathcal{Q}=\mathfrak{Q}$. On the other hand if $\mathcal{Q}$ is a finite set whose elements satisfy some separation condition and their number is independent of $n$ the assertions of Theorem 1 hold.

The second problem studied in the paper is the minimax adaptive estimation with respect to the parameter $(\beta, L)$. Let $\mathcal{Q} \subseteq \mathfrak{Q}$ be fixed and let

$$
\varphi_{n}(\beta, L)=\inf _{\widetilde{f}} \sup _{\mathcal{F}(\beta, L, \mathcal{Q})} \mathcal{R}_{n}^{(p)}[\tilde{f}, f], \quad \beta>0, L>0 .
$$

Our objective is to answer on the following question: does there exist an estimator $f^{*}$ such that

$$
\limsup _{n \rightarrow \infty} \varphi_{n}^{-1}(\beta, L) \sup _{\mathcal{F}(\beta, L, \mathcal{Q})} \mathcal{R}_{n}^{(p)}\left[f^{*}, f\right]<\infty, \quad \forall \beta>0, L>0 ? .
$$

We will prove that the answer is positive if $\mathcal{Q}$ is a net in $\mathfrak{Q}$ satisfying some separation condition and $\beta \in(0, \mathfrak{b}]$, where $\mathfrak{b}>0$ is an arbitrary but a priori chosen number.

Historical notes There is a vast literature dealing with minimax and minimax adaptive density estimation. The interested reader can find very detailed overview on this topic in Lepski (2015). As it was saying above, we will follow the modeling strategy which consists in imposing additional structural assumptions on the function to be estimated. This approach was pioneered by Stone (1985) who discussed the trade-off between flexibility and dimensionality of nonparametric models and formulated the heuristic dimensionality reduction principle. Standard examples of structural nonparametric models are single-index, additive, projection pursuit or multi-index model, composite functions structure etc. The minimax and minimax adaptive results in these models (mostly in the nonparametric regression context) were

imsart-generic ver. 2014/10/16 file: rotation-arXiv.tex date: February 26, 2020 
obtain in Huber (1985), Chen (1991) Golubev (1992), Hristache et al (2001), Horowitz and Mammen (2007), Juditsky, Lepski and Tsybakov (2009), Goldenshluger and Lepski (2009), Lepski and Serdyukova (2014) among many others. However, when one is talking about the multivariate density estimation there are not so many articles where minimax and minimax adaptive results were obtained. The problems and models similar to those considered in the present paper were studied in Samarov and Tsybakov (2007), Amato et al (2010), Lepski (2013), Rebelles (2015a), Rebelles (2015b). We would like especially to mention the paper Samarov and Tsybakov (2004) where $d$-dimensional variant of our model was considered. Some problems in this article have been studied under pointwise risk and we will provide a detailed comparison of them and our results after Theorem 4 .

Definitions, assumptions and notations For any $\mathcal{Q} \in \mathfrak{Q}$ and any function $f \in \mathcal{F}(\beta, L, \mathcal{Q})$ we denote by $\boldsymbol{Q}_{f} \in \mathcal{Q}$ and $\boldsymbol{g}_{f} \in \mathcal{G}(\beta, L)$ the quantities obtained from the relation

$$
f(\bullet)=g_{f}\left(\boldsymbol{Q}_{f} \bullet\right) .
$$

Obviously this representation is not unique and later on we consider an arbitrary couple $\left(\boldsymbol{Q}_{f}, \boldsymbol{g}_{f}\right)$ for which the latter relation holds.

Furthermore $\|\cdot\|_{\infty}$ will be used for the supremum norm on $\mathbb{R}$, the integer part of $a>0$ will be denoted by $\lfloor a\rfloor$ and any $Q \in \mathfrak{Q}$ will be presented as

$$
Q=\left(q, q_{\perp}\right)=\left(\begin{array}{cc}
q_{1} & -q_{2} \\
q_{2} & q_{1}
\end{array}\right) .
$$

Definition 2. Let $\beta=r+\alpha, r \in \mathbb{N}, 0<\alpha \leq 1$ and $L>0$ be given. We say that $w: \mathbb{R} \rightarrow \mathbb{R}$ belongs to the Hölder class $\mathbb{H}(\beta, L)$ if it is $r$-times continuously differentiable, $\left\|w^{(j)}\right\|_{\infty} \leq L$ for any $j=0, \ldots, r$ and

$$
\left\|w^{(r)}(\cdot+\mathfrak{z})-w^{(r)}(\cdot)\right\|_{\infty} \leq\left.\left. L\right|_{\mathfrak{z}}\right|^{\alpha}, \quad \forall \mathfrak{z} \in \mathbb{R} .
$$

For given $\mathfrak{b} \geq 1$ we denote by $\mathbb{K}_{\mathfrak{b}}$ the set of functions $\mathcal{K}: \mathbb{R} \rightarrow \mathbb{R}$ satisfying the following assumption.

Assumption 1. $\mathcal{K} \in \mathbb{L}_{1}(\mathbb{R}) \cap \mathbb{L}_{\infty}(\mathbb{R}), \int_{\mathbb{R}} \mathcal{K}(u) \mathrm{d} u=1$ and

$$
\int_{\mathbb{R}} \mathcal{K}(u) u^{j} \mathrm{~d} u=0, j=1, \ldots, 2\lfloor\mathfrak{b}\rfloor, \quad \int_{\mathbb{R}}|\mathcal{K}(t)||t|^{2 \mathfrak{b}} \mathrm{d} t<\infty .
$$

With any $\mathcal{K} \in \mathbb{K}_{\mathfrak{b}}$ we associate the following quantity:

$$
C(\mathcal{K}, \mathfrak{b}, \mathbf{s})=\sup _{b \leq \mathfrak{b}} \int_{\mathbb{R}^{2}}\left|\mathcal{K}\left(t_{1}\right) \mathcal{K}\left(t_{2}\right)\right|\left[\mathbf{s}\left(t_{1}^{2}+t_{2}^{2}\right)^{b}+1\right]^{2} \mathrm{~d} t_{1} \mathrm{~d} t_{2}, \mathbf{s}>0 .
$$

For any $D, Q \in \mathfrak{Q}$ we will write

$$
p_{1}:=p_{1}(D, Q)=q^{T} d_{\perp}, \quad p_{2}:=p_{2}(D, Q)=q^{T} d
$$


and set $\varrho(D, Q):=\min \left[\left|p_{1}(Q, D)\right|,\left|p_{2}(D, Q)\right|\right]$.

For given $\delta>0$ we denote by $\mathbb{Q}_{\delta}$ the set of all subsets of $\mathfrak{Q}$ consisting of $\delta$-distinguishable points with respect to $\varrho$. Recall that $Q_{1}, Q_{2}$ are called $\delta$-distinguishable with respect to $\varrho$ if $\varrho\left(Q_{1}, Q_{2}\right) \geq \delta$. For any $\mathcal{Q}_{\delta} \in \mathbb{Q}_{\delta}$ let

$$
\mathfrak{n}\left(\mathcal{Q}_{\delta}\right)=\ln \left(\operatorname{card}\left(\mathcal{Q}_{\delta}\right)\right) .
$$

Remark 1. We note that $p_{1}(D, Q)=-p_{1}(Q, D), p_{2}(D, Q)=p_{2}(Q, D)$ and $p_{1}(Q, Q)=0$. Additionally it can be easily checked that

$$
\varrho\left(Q_{1}, Q_{3}\right) \leq 2 \sqrt{2}\left[\varrho\left(Q_{1}, Q_{2}\right)+\varrho\left(Q_{2}, Q_{3}\right)\right], \quad \forall Q_{1}, Q_{2}, Q_{3} \in \mathfrak{Q} .
$$

Hence, we assert that $\varrho$ is a $2 \sqrt{2}$-pseudo-inframetrics on $\mathfrak{Q}$.

If $\mathfrak{Q}_{\delta} \in \mathbb{Q}_{\delta}$ is the maximal $\delta$-net of $\mathfrak{Q}$ in $\varrho$ then $\mathfrak{n}_{\delta}:=\mathfrak{n}\left(\mathfrak{Q}_{\delta}\right)$ is called $\delta$ capacity of $\mathfrak{Q}$. Recall that the $\delta$-capacity (as well as the $\delta$-entropy) is used for classifying compact metric sets according to their massivity.

From now on $\delta \in(0,1)$ (possibly dependent on $n$ ) is assumed to be fixed and the number of observations $n \geq 3$.

\section{Main results}

In this section we develop the minimax and adaptive minimax theories over collection of functional classes $\mathcal{F}\left(\beta, L, \mathcal{Q}_{\delta}\right), \mathcal{Q}_{\delta} \in \mathbb{Q}_{\delta}$.

\subsection{Lower bounds}

We start with presenting two lower bound results.

Theorem 2. For any $\beta_{1}>0, \beta_{2}>0$ and $p \geq 1$ there exists $\mathbf{c}_{1}>0$ such that for any $Q \in \mathfrak{Q}$ and $L>0$

$$
\liminf _{n \rightarrow \infty} \inf _{\widetilde{f}} \sup _{\beta \in\left\{\beta_{1}, \beta_{2}\right\}}\left(L^{\frac{2}{2 \beta+1}} \ln (n) / n\right)^{-\frac{\beta}{2 \beta+1}} \sup _{\mathcal{F}(\beta, L,\{\boldsymbol{Q}\})} \mathcal{R}_{n}^{(p)}[\tilde{f}, f] \geq \mathbf{c}_{1} .
$$

For any $\beta>0, L>0$ and $p \geq 1$ there exists $\mathbf{c}_{2}>0$ such that for an arbitrary sequence $\delta_{n}>0$ satisfying $\delta_{n} \geq(\ln (n))^{\frac{2 \beta+2}{2 \beta+1}}\left(L^{-2} / n\right)^{\frac{1}{2 \beta+1}}$ and any $\mathcal{Q}_{\delta_{n}} \in \mathbb{Q}_{\delta_{n}}$ one has

$$
\liminf _{n \rightarrow \infty}\left(\mathfrak{n}\left(\mathcal{Q}_{\delta_{n}}\right) / n\right)^{-\frac{\beta}{2 \beta+1}} \inf _{\widetilde{f}} \sup _{\mathcal{F}\left(\beta, L, \mathcal{Q}_{\delta}\right)} \mathcal{R}_{n}^{(p)}[\tilde{f}, f] \geq \mathbf{c}_{2} .
$$

Some remarks are in order.

$\mathbf{1}^{\mathbf{0}}$. The first assertion of the theorem is quite standard and its proof will be omitted. The fact that $\boldsymbol{Q}$ is known reduces the considered problem to adaptive poinwise estimation over collection of Hölder classes under 
independent hypothesis (it suffices to consider new observation sequence $\left.\boldsymbol{Q}^{T} X_{1}, \ldots, \boldsymbol{Q}^{T} X_{n}\right)$. Then (2.1) follows, in particular, from the lower bound result obtained in Rebelles (2015a). As usual, see for instance Rebelles (2015a), there is $\ln (n)$-price to pay for adaptation. That means that minimax result given in Theorem 1 differs from whose in (2.1) by $\ln (n)$-factor.

$\mathbf{2}^{\mathbf{0}}$. The proof of the second assertion is much more involved. If $\delta=$ constant then the factor $\mathfrak{n}\left(\mathcal{Q}_{\delta}\right)$ can be viewed as the price to pay for structural adaptation (with respect to unknown rotation $\boldsymbol{Q}_{f} \in \mathcal{Q}_{\delta}$ ). However this is a constant factor, the asymptotics of minimax risk with respect to $n$ remains the same and coincides with whose in Theorem 1 . The situation changes completely if $\delta=\delta_{n} \rightarrow 0, n \rightarrow \infty$. Indeed, if $\mathfrak{n}\left(\mathcal{Q}_{\delta_{n}}\right) \rightarrow \infty$ the minimax rate found in Theorem 1 is no more achievable and $\mathfrak{n}\left(\mathcal{Q}_{\delta_{n}}\right)$ is the minimal price to pay for structural adaptation over $\mathcal{Q}_{\delta_{n}}$. It is not difficult to see that for any $\mathcal{Q}_{\delta_{n}} \in \mathbb{Q}_{\delta_{n}}$

$$
\mathfrak{n}\left(\mathcal{Q}_{\delta_{n}}\right) \leq \mathfrak{n}\left(\mathfrak{Q}_{\delta_{n}}\right) \asymp\left|\ln \left(\delta_{n}\right)\right|, n \rightarrow \infty .
$$

It yields in particular that if $\delta_{n} \sim n^{-a}$ for some $a>0$, then the minimal price to pay for structural adaptation on $\mathfrak{Q}_{\delta_{n}}$ is proportional to $\ln (n)$.

\subsection{Pointwise selection rules}

Our estimation procedures are based on the original selection rule from the family of kernel-type estimators. One of them called adaptive selection rule is inspired by general approach discussed in Goldenshluger and Lepski (2012) but the procedure is completely new.

Family of estimators. For any $\mathcal{K}$ satisfying Assumption 1 and $h>0$ denote $\mathcal{K}_{h}(\cdot)=h^{-1} \mathcal{K}(\cdot / h)$. For any $D \in \mathfrak{Q}$ and $x \in \mathbb{R}^{2}$ introduce the estimator

$$
\widetilde{f}_{h, D}(x)=\left[n^{-1} \sum_{k=1}^{n} \mathcal{K}_{h}\left(d^{T}\left(X_{k}-x\right)\right)\right]\left[n^{-1} \sum_{k=1}^{n} \mathcal{K}_{h}\left(d_{\perp}^{T}\left(X_{k}-x\right)\right)\right] .
$$

Set $\mathcal{H}=\left\{e^{-k}, k=0,1, \ldots,\lfloor\ln (n)\rfloor\right\}$ and let $\mathcal{Q}_{\delta} \in \mathbb{Q}_{\delta}$ be given. Introduce the following estimator's family:

$$
\mathfrak{F}\left(\mathcal{Q}_{\delta}, \mathcal{H}\right)=\left\{\widetilde{f}_{h, D}(x), D \in \mathcal{Q}_{\delta}, h \in \mathcal{H}\right\} .
$$

It is worth noting that if $\mathcal{Q}_{\delta}=\{D\}$ the estimator $\widetilde{f}_{h, D}(x)$ is exactly the estimator $\widehat{g}\left(D^{T} x\right), \widehat{g}(x)=\widehat{g}_{1}\left(x_{1}\right) \widehat{g}_{2}\left(x_{2}\right)$ introduced in the discussion preceded Theorem 1.

Below we propose two different data-driven selection rules from this collection. The first one, called below adaptive selection rule, will be used in

imsart-generic ver. 2014/10/16 file: rotation-arXiv.tex date: February 26, 2020 
the situation when the parameters $\beta, L$ are unknown, $\mathcal{Q}_{\delta_{n}}$ is an arbitrary element of $\mathbb{Q}_{\delta_{n}}$ with any $\delta_{n}>0$ satisfying

$$
\delta_{n} \geq(\ln (n) / n)^{\frac{1}{4 \mathfrak{b}+2}} .
$$

Here $\mathfrak{b} \geq 1$ is an arbitrary but a priori chosen number and $\beta \in(0, \mathfrak{b}]$.

The second one, called minimax selection rule, will be applied when $\beta, L$ are known. The interesting case here is $\delta=$ constant for example $\operatorname{card}\left(\mathcal{Q}_{\delta}\right)=2$. Another intriguing case is $\delta=\delta_{n}$ such that $\mathfrak{n}\left(\mathcal{Q}_{\delta_{n}}\right)=o(\ln (n)), n \rightarrow \infty$.

Auxiliary estimator $\operatorname{Set} K_{h}(t)=\mathcal{K}_{h}\left(t_{1}\right) \mathcal{K}_{h}\left(t_{2}\right), t \in \mathbb{R}^{2}, h>0$,

$$
\Gamma=\left(\begin{array}{cc}
1 & 0 \\
0 & -1
\end{array}\right), \quad \Omega=\left(\begin{array}{ll}
0 & 1 \\
1 & 0
\end{array}\right),
$$

and define for any $D, Q \in \mathfrak{Q}$ what we will call the auxiliary estimator

$$
\bar{f}_{h,(D, Q)}(x)=\frac{1}{n(n-1)} \sum_{k, l=1, k \neq l}^{n} K_{h}\left(p_{1} \Omega \Gamma X_{k}+p_{2} X_{l}-\Omega \Gamma Q D \Omega x\right) .
$$

Remark that $\bar{f}_{h,(D, Q)}$ is a $U$-statistics of the order 2 if $\varrho(D, Q) \neq 0$. Put also

$$
\tilde{f}_{h,(D, Q)}(x)=\left\{\begin{array}{cc}
\bar{f}_{h,(D, Q)}(x), & D \neq Q \\
\widetilde{f}_{h, Q}(x), & D=Q .
\end{array}\right.
$$

\subsubsection{Adaptive selection rule}

Let $\mathbf{A}>0$ be a constant given in section 3.2.1.

Set $\mathfrak{H}=\left\{h \in \mathcal{H}: 1 / \ln (\ln (n)) \geq h \geq[\ln (n)]^{2} / n\right\}$ and

$$
\widehat{\mathcal{U}}_{n}=\sup _{\eta \in \mathfrak{H}} \sup _{D \in \mathcal{Q}_{\delta_{n}}} \sup _{b \in\left\{d, d_{\perp}\right\}}\left\{1 \vee\left[n^{-1} \sum_{k=1}^{n}\left|\mathcal{K}_{\eta}\left(b^{T}\left(X_{k}-x\right)\right)\right|\right]^{2}\right\} .
$$

Introduce for any $Q \in \mathcal{Q}_{\delta}$ and any $h \in \mathfrak{H}$

$$
R_{n}(Q, h)=\sup _{\substack{\eta, \eta^{\prime} \in \mathfrak{S} ; \\ \eta^{\prime} \leq \eta \leq h}} \sup _{D \in \mathcal{Q}_{\delta_{n}}}\left[\left|\widetilde{f}_{\eta,(D, Q)}(x)-\widetilde{f}_{\eta^{\prime}, D}(x)\right|-\mathbf{A} \widehat{\mathcal{U}}_{n}\left(\frac{\ln (n)}{n \eta^{\prime}}\right)^{1 / 2}\right]_{+}
$$

and define

$$
(\widehat{h}, \widehat{Q})=\arg \min _{Q \in \mathcal{Q}_{\delta_{n}}, h \in \mathfrak{H}}\left[R_{n}(Q, h)+\mathbf{A} \widehat{\mathcal{U}}_{n} \sqrt{\ln (n) / n h}\right]
$$

The suggested estimator is then $\widehat{f}=\widetilde{f}_{\widehat{h}, \widehat{Q}}(x)$. 
Theorem 3. Let $p \geq 1, \mathfrak{b} \geq 1$ and $\mathcal{K} \in \mathbb{K}_{\mathfrak{b}}$ be fixed. Then for any $\beta_{1}, \beta_{2} \in$ $(0, \mathfrak{b}], L>0$, any $\delta_{n}$ satisfying (2.3) and any $\mathcal{Q}_{\delta_{n}} \in \mathbb{Q}_{\delta_{n}}$ one has

$$
\limsup _{n \rightarrow \infty} \sup _{\beta \in\left\{\beta_{1}, \beta_{2}\right\}}\left(L^{2 / \beta} \ln (n) / n\right)^{-\frac{\beta}{2 \beta+1}} \sup _{\mathcal{F}\left(\beta, L, \mathcal{Q}_{\delta_{n}}\right)} \mathcal{R}_{n}^{(p)}[\widehat{f}, f]<\infty .
$$

We conclude that the estimator $\widehat{f}$ provides the optimal (in view of the first assertion of Theorem 2) accuracy of estimation simultaneously over the collection of functional classes $\mathcal{F}\left(\beta, L, \mathcal{Q}_{\delta_{n}}\right)$.

\subsection{Minimax selection rule}

As it has been already mentioned the construction of the minimax estimator is much more complicated. In particular it requires non-trivial splitting of the observation sequence in order to get desirable theoretical results. However the implementation of our minimax procedure for reasonable sample size does not require such splitting, see remark after Theorem 4 .

Let $\beta>0, L>0, \delta_{n}>0, \mathcal{Q}_{\delta_{n}} \in \mathbb{Q}_{\delta_{n}}$ be given. Introduce the following notations. Set $\ell_{\mathbf{0}}=\ln (n)$ and let for any $\mathbf{i} \in \mathbb{N}^{*}$

$$
\begin{aligned}
& \ell_{\mathbf{i}}=\ln \left(\ell_{\mathbf{i}-1}\right), \quad \omega_{\mathbf{i}}=\ell_{\mathbf{i}} \vee 4+\mathfrak{n}\left(\mathcal{Q}_{\delta_{n}}\right) \\
& \mathbf{i}^{*}=\min \left\{\mathbf{i} \in \mathbb{N}^{*}: \omega_{\mathbf{i}}=4+\mathfrak{n}\left(\mathcal{Q}_{\delta_{n}}\right)\right\} .
\end{aligned}
$$

Set also for any $\mathbf{i}=1, \ldots \mathbf{i}^{*}-1$

$$
\mathbf{n}_{\mathbf{i}}=\left\lfloor n \ell_{\mathbf{i}}^{-1}\right\rfloor, \quad N_{\mathbf{i}}=\lfloor n / 4\rfloor+\sum_{\mathbf{j}=1}^{\mathbf{i}} \mathbf{n}_{\mathbf{j}} .
$$

Remark 2. By definition $\ell_{\mathbf{i}^{*}-1} \geq 4$ that obviously implies that $\ell_{\mathbf{i}^{*}-j} \geq 4^{j}$ for any $j=1, \ldots, \mathbf{i}^{*}-1$. Hence

$$
N_{\mathbf{i}^{*}-1} \leq\lfloor n / 4\rfloor+n \sum_{\mathbf{i}=1}^{\mathbf{i}^{*}-1} \ell_{\mathbf{i}}^{-1}<3 n / 4 .
$$

In view of the latter remark introduce the following splitting of the observation sequence. For any $\mathbf{i}=1, \ldots \mathbf{i}^{*}-1$ set

$X^{(\mathbf{i})}=X_{N_{\mathbf{i}-1}+1}, \ldots, X_{N_{\mathbf{i}}}, X^{\left(\mathbf{i}^{*}\right)}=X_{N_{\mathbf{i}^{*}-1}+1}, \ldots, X_{n}, X^{(\mathbf{0})}=X_{1}, \ldots, X_{\lfloor n / 4\rfloor}$.

We remark that $X^{(\mathbf{i})}, \mathbf{i}=0, \ldots \mathbf{i}^{*}$, are mutually independent and later on all objects measurable with respect to $X^{(\mathbf{i})}, \mathbf{i}=1, \ldots \mathbf{i}^{*}$ will be marked by "(i) ". Put $\mathbf{n}_{\mathbf{i}^{*}}=n-N_{\mathbf{i}^{*}-1}$ and for any $\mathbf{i}=1, \ldots \mathbf{i}^{*}$ introduce $h_{\mathbf{i}}=\left(L^{-4} \omega_{\mathbf{i}} / \mathbf{n}_{\mathbf{i}}\right)^{\frac{1}{2 \beta+1}}$,

$$
\begin{aligned}
R_{n}^{(\mathbf{i})}(Q) & =\sup _{D \in \mathcal{Q}_{\delta_{n}}}\left[\left|\widetilde{f}_{h_{\mathbf{i}},(D, Q)}^{(\mathbf{i})}(x)-\widetilde{f}_{h_{\mathbf{i}}, D}^{(\mathbf{i})}(x)\right|-\mathbf{B} L^{2} h_{\mathbf{i}}^{\beta}\right]_{+} ; \\
\widehat{Q}^{(\mathbf{i})} & =\arg \min _{Q \in \mathcal{Q}_{\delta}} R_{n}^{(\mathbf{i})}(Q), \quad \widehat{f}^{(\mathbf{i})}(x)=\widetilde{f}_{h_{\mathbf{i}}, \widehat{Q}^{(\mathbf{i})}}^{(\mathbf{i})}(x) .
\end{aligned}
$$

imsart-generic ver. 2014/10/16 file: rotation-arXiv.tex date: February 26, 2020 
where $\mathbf{B}>0$ is a constant given in section 3.2.2.

Let $\widehat{f}(x)$ be the estimator from Theorem 3 corresponding to the choice $\mathfrak{b}=\beta$ and constructed from $X^{(\mathbf{0})}$. Define for any $\mathbf{i}=1, \ldots \mathbf{i}^{*}$

$$
\breve{f}^{(\mathbf{0})}(x)=\widehat{f}(x), \quad \breve{f}^{(\mathbf{i})}(x)= \begin{cases}\breve{f}^{(\mathbf{i}-1)}(x), & R_{n}^{(\mathbf{i})}\left(\widehat{Q}^{(\mathbf{i})}\right) \neq 0 ; \\ \widehat{f}^{(\mathbf{i})}(x), & R_{n}^{(\mathbf{i})}\left(\widehat{Q}^{(\mathbf{i})}\right)=0 .\end{cases}
$$

The suggested estimator is then $\breve{f}=\breve{f}^{\left(\mathbf{i}^{*}\right)}(x)$.

Theorem 4. Let $p \geq 1, \beta>0, L>0, \delta_{n}$ satisfying (2.3) with $\mathfrak{b}=\beta$, $\mathcal{Q}_{\delta_{n}} \in \mathbb{Q}_{\delta_{n}}$ and $\mathcal{K} \in \mathbb{K}_{\beta}$ be fixed. Then

$$
\left.\limsup _{n \rightarrow \infty}\left(L^{\frac{2}{\beta}} \mathfrak{n}\left(\mathcal{Q}_{\delta_{n}}\right) / n\right)\right)^{-\frac{\beta}{2 \beta+1}} \sup _{\mathcal{F}\left(\beta, L, \mathcal{Q}_{\delta_{n}}\right)} \mathcal{R}_{n}^{(p)}[\breve{f}, f]<\infty .
$$

$\mathbf{1}^{\mathbf{0}}$. To the best of our knowledge the construction led to the estimator $\breve{f}$ has no analogue in the existing literature on the minimax and minimax adaptive estimation. Although formally $\mathbf{i}^{*} \rightarrow \infty, n \rightarrow \infty$, but $\mathbf{i}^{*}=1$ for $n=10^{100}$ and for any $\mathcal{Q}_{\delta} \in \mathfrak{Q}_{\delta}$ such that $\mathfrak{n}\left(\mathcal{Q}_{\delta}\right) \geq 6$. It worth noting that if $\mathbf{i}^{*}$ is independent of $n$ (what is the case for many sequences $\delta_{n} \rightarrow 0$ ) the splitting of data is not needed anymore. The estimator construction remains the same but the estimators $\widehat{f}^{(\mathbf{i})}, \mathbf{i}=1, \ldots, \mathbf{i}^{*}$ are built from the whole data set. The proof of the minimax optimality of this procedure is the simple modification of the proof of Theorem 4 and is left to an interested reader.

$2^{\mathbf{0}}$. Comparing the results presented in the second assertion of Theorem 2 and in Theorem 4 we conclude that $\breve{f}$ is minimax optimal on $\mathcal{F}\left(\beta, L, \mathcal{Q}_{\delta_{n}}\right)$. In particular if $\delta>0$ is independent of $n$ there is only a constant factor to be paid for the adaptation w.r.t unknown rotation. On the other hand if $\left.\delta_{n} \asymp n^{-a}, a \leq[4 \beta+2)\right]^{-1}$ and $\mathfrak{n}\left(\mathcal{Q}_{\delta_{n}}\right) \asymp \ln (n)$ for instance $\mathcal{Q}_{\delta_{n}}=\mathfrak{Q}_{\delta_{n}}$, cf. (2.3), the adaptive estimator from Theorem 3 with $\mathfrak{b}=\beta$ is minimax optimal as well. We remark that this estimator does not require any splitting of the observations.

$\mathbf{3}^{\mathbf{0}}$. In Samarov and Tsybakov (2004) the authors studied the same observation model but in an arbitrary dimension $d \geq 2$. Their estimation procedure is based on the completely different principles. First, they estimate the unknown rotation matrix and then plug-in it to the estimator $\widehat{f}_{\mathfrak{h}, \bullet}$. It is worth noting that the estimation of the rotation (the important problem itself) requires very restrictive assumptions. In particular the authors assumes that $\beta>5$ if $d=2$ and that the observations possess finite absolute moment of order 4 . We impose none of these assumptions. Although Samarov and Tsybakov (2004) it is assumed that the marginal densities belong to Hölder class the obtained rate of convergence is not uniform one. In particular the authors established the rate which is the same as in Theorem 
1 which, in view of the lower bound of Theorem 2, is possible if and only if the number of rotations is finite and independent of $n$. We think that the use of plug-in approach in structural models is either too restrictive or not optimal. It seems that the technique of structural adaptation is much more adequate for such kind of problems.

\section{Proofs of Theorems 2-4}

Recall that we will proof only the second assertion of Theorem 2.

\subsection{Proof of the second assertion of Theorem 2}

To simplicity of notation we will prove the theorem for $x=0$. The transition to the general case does not bring any additional difficulty.

$\mathbf{1}^{\mathbf{0}}$. Let $\mathbf{n}(y)=\frac{1}{\sqrt{2 \pi \sigma^{2}}} e^{-\frac{y^{2}}{2 \sigma}}$, where $\sigma^{2}>0$ is chosen in order to guarantee $\mathbf{n}(\cdot) \in \mathbb{H}(\beta, L / 2)$. Let $\lambda: \mathbb{R} \rightarrow \mathbb{R}$ be a symmetric function satisfying

$$
\lambda \in \mathbb{H}(\beta, 1 / 2), \quad \int_{\mathbb{R}} \lambda(y) \mathrm{d} y=0, \quad \lambda(0)>0, \quad \lambda(y)=0, \forall y \notin[-1,1] .
$$

Let $\varpi>0$ be a constant the choice of which will be done later. Set $\varepsilon=$ $\left(\varpi L^{-2} \mathfrak{n}\left(\mathcal{Q}_{\delta_{n}}\right) / n\right)^{\frac{1}{2 \beta+1}}$ and let

$$
\mathrm{p}(y)=\mathbf{n}(y)+L \varepsilon^{\beta} \lambda(y / \varepsilon), \quad y \in \mathbb{R} .
$$

Obviously, $\mathrm{p} \in \mathbb{H}(\beta, L), \int_{\mathbb{R}} \mathrm{p}(y) \mathrm{d} y=1$ and for all $n$ large enough $\mathrm{p}>0$. Hence $\mathrm{p}$ is a probability density. Define

$$
g(x)=\mathrm{p}\left(x_{1}\right) \mathrm{p}\left(x_{2}\right), \quad \mathbf{N}(x)=\mathbf{n}\left(x_{1}\right) \mathbf{n}\left(x_{2}\right), x=\left(x_{1}, x_{2}\right) \in \mathbb{R}^{2} .
$$

We can assert that $g, \mathbf{N} \in \mathcal{G}(\beta, L)$. Let $\mathcal{Q}_{\delta_{n}}=\left\{Q_{0}, \ldots Q_{\mathfrak{m}_{n}}\right\}$, where we have denoted $\mathfrak{m}_{n}=\exp \left\{\mathfrak{n}\left(\mathcal{Q}_{\delta_{n}}\right)\right\}-1=\operatorname{card}\left(\mathcal{Q}_{\delta_{n}}\right)-1$. Set finally

$$
f_{0}(\bullet)=\mathbf{N}(\bullet), \quad f_{j}(\bullet)=g\left(Q_{j}^{T} \bullet\right), \quad j=1, \ldots, \mathfrak{m}_{n} .
$$

We assert that $\left\{f_{j}, j=0, \ldots, \mathfrak{m}_{n}\right\} \subset \mathcal{F}\left(\beta, L, \mathcal{Q}_{\delta_{n}}\right)$. Here we have used that $\mathbf{N}(\bullet) \equiv \mathbf{N}\left(Q_{0}^{T} \bullet\right)$. Additionally, for any $j=1, \ldots, \mathfrak{m}_{n}$

$$
\left|f_{j}(0)-f_{0}(0)\right| \mid=\left(\mathbf{n}(0)+L \lambda(0) \varepsilon^{\beta}\right)^{2}-\mathbf{n}^{2}(0) \geq c\left(\varpi L^{\frac{1}{\beta}} \mathfrak{n}\left(\mathcal{Q}_{\delta_{n}}\right) / n\right)^{\frac{\beta}{2 \beta+1}},
$$

for all $n$ large enough. Here $c>0$ is a numerical constant independent on $n$ and $L$. Introduce

$$
Z_{n}=\frac{1}{\mathfrak{m}_{n}} \sum_{j=1}^{\mathfrak{m}_{n}} \prod_{i=1}^{n} \frac{f_{j}\left(X_{i}\right)}{f_{0}\left(X_{i}\right)}
$$

imsart-generic ver. 2014/10/16 file: rotation-arXiv.tex date: February 26, 2020 
In view of (3.1) and in accordance with Corollary 2 of Proposition 5 in Kerkyacharian et al. (2007) the assertion of the theorem will follow with $\mathbf{c}_{2}=2^{-p}\left(1-\sqrt{\frac{\Upsilon+1}{\Upsilon+5}}\right)$ if we prove that

$$
\Upsilon:=\limsup _{n \rightarrow \infty} \mathbb{E}_{f_{0}}\left\{Z_{n}^{2}\right\}<\infty
$$

$2^{0}$. We have

$$
\begin{aligned}
& \mathbb{E}_{f_{0}}\left\{Z_{n}^{2}\right\}=\frac{1}{\mathfrak{m}_{n}^{2}} \sum_{j=1}^{\mathfrak{m}_{n}}\left(\int_{\mathbb{R}^{2}} \frac{f_{j}^{2}(x)}{f_{0}} \mathrm{~d} x\right)^{n}+\frac{1}{\mathfrak{m}_{n}^{2}} \sum_{\substack{k, j=1 \\
k \neq j}}^{\mathfrak{m}_{n}}\left(\int_{\mathbb{R}^{2}} \frac{f_{j}(x) f_{k}(x)}{f_{0}} \mathrm{~d} x\right)^{n} \\
& \leq \mathfrak{m}_{n}^{-1} \sup _{j=1, \ldots, \mathfrak{m}_{n}}\left(\int_{\mathbb{R}^{2}} \frac{f_{j}^{2}(x)}{f_{0}} \mathrm{~d} x\right)^{n}+\sup _{\substack{k, j=1, \ldots, \mathfrak{m}_{n} \\
k \neq j}}\left(\int_{\mathbb{R}^{2}} \frac{f_{j}(x) f_{k}(x)}{f_{0}} \mathrm{~d} x\right)^{n} .
\end{aligned}
$$

$\mathbf{2}^{\mathbf{0}} \mathbf{a}$. Denote by $\mathbf{M}_{\varepsilon}(\cdot)=\mathbf{M}_{1}(\cdot)+\mathbf{M}_{2}(\cdot)+L \varepsilon^{\beta} \Lambda(\cdot)$, where we put

$$
\mathbf{M}_{1}(x)=\mathbf{n}\left(x_{1}\right) \lambda\left(x_{2} / \varepsilon\right), \quad \mathbf{M}_{2}(x)=\lambda\left(x_{1} / \varepsilon\right) \mathbf{n}\left(x_{2}\right), \quad \Lambda=\lambda\left(x_{1} / \varepsilon\right) \lambda\left(x_{2} / \varepsilon\right) .
$$

Since $\int \lambda=0$ we have

$$
\int_{\mathbb{R}^{2}} \mathbf{M}_{\varepsilon}\left(Q_{j}^{T} x\right) \mathrm{d} x=0, \quad \forall j=1, \ldots, \mathfrak{m}_{n} .
$$

Note also that for any $k, j=1, \ldots, \mathfrak{m}_{n}$

$$
\begin{gathered}
f_{j}(x) f_{k}(x)=\left[\mathbf{N}(x)+L \varepsilon^{\beta} \mathbf{M}_{\varepsilon}\left(Q_{j}^{T} x\right)\right]\left[\mathbf{N}(x)+L \varepsilon^{\beta} \mathbf{M}_{\varepsilon}\left(Q_{k}^{T} x\right)\right] \\
=\mathbf{N}^{2}(x)+L \varepsilon^{\beta} \mathbf{N}(x)\left[\mathbf{M}_{\varepsilon}\left(Q_{j}^{T} x\right)+\mathbf{M}_{\varepsilon}\left(Q_{k}^{T} x\right)\right]+L^{2} \varepsilon^{2 \beta} \mathbf{M}_{\varepsilon}\left(Q_{j}^{T} x\right) \mathbf{M}_{\varepsilon}\left(Q_{k}^{T} x\right) .
\end{gathered}
$$

Thus, in view of (3.4) we have for any $j, k=1, \ldots, \mathfrak{m}_{n}$

$$
a_{j, k}:=\int_{\mathbb{R}^{2}} \frac{f_{j}(x) f_{k}(x)}{f_{0}(x)} \mathrm{d} x=1+L^{2} \varepsilon^{2 \beta} \int_{\mathbb{R}^{2}} \frac{\mathbf{M}_{\varepsilon}\left(Q_{j}^{T} x\right) \mathbf{M}_{\varepsilon}\left(Q_{k}^{T} x\right)}{\mathbf{N}(x)} \mathrm{d} x .
$$

$2^{0}$ b. It yields first,

$$
\begin{aligned}
a_{j, j} & =1+L^{2} \varepsilon^{2 \beta} \int_{\mathbb{R}^{2}} \frac{\mathbf{M}_{\varepsilon}^{2}(x)}{\mathbf{N}(x)} \mathrm{d} x \\
& \leq 1+3 L^{2} \varepsilon^{2 \beta}\left[\int_{\mathbb{R}^{2}} \frac{\mathbf{M}_{1}^{2}(x)}{\mathbf{N}(x)} \mathrm{d} x+\int_{\mathbb{R}^{2}} \frac{\mathbf{M}_{2}^{2}(x)}{\mathbf{N}(x)} \mathrm{d} x+L^{2} \varepsilon^{2 \beta} \int_{\mathbb{R}^{2}} \frac{\Lambda^{2}(x)}{\mathbf{N}(x)} \mathrm{d} x\right] \\
& =1+3 L^{2} \varepsilon^{2 \beta}\left[2 \int_{\mathbb{R}} \frac{\lambda^{2}(y / \varepsilon)}{\mathbf{n}(y)} \mathrm{d} y+L^{2} \varepsilon^{2 \beta}\left(\int_{\mathbb{R}} \frac{\lambda^{2}(y / \varepsilon)}{\mathbf{n}(y)} \mathrm{d} y\right)^{2}\right] .
\end{aligned}
$$

imsart-generic ver. 2014/10/16 file: rotation-arXiv.tex date: February 26, 2020 
From now on we will assume that $n$ is sufficiently large to guarantee that $\mathbf{n}(y) \geq 2^{-1} \mathbf{n}(0)$ for all $y \in[-\varepsilon, \varepsilon]$. Then, taking into account that $\lambda(y / \varepsilon)=0$ for any $y \notin[-\varepsilon, \varepsilon]$ we obtain for all $n$ large enough

$$
a_{j, j} \leq 1+C_{1} L^{2} \varepsilon^{2 \beta+1} .
$$

where $C_{1}$ is independent on $n$ and $L$. Hence, choosing $\varpi=C_{1}^{-1}$ we get

$$
a_{j, j}^{n} \leq e^{n C_{1} L^{2} \varepsilon^{2 \beta+1}}=e^{C_{1} \varpi \mathfrak{n}\left(\mathcal{Q}_{\delta_{n}}\right)}=\mathfrak{m}_{n}, \quad \forall j=1, \ldots, \mathfrak{m}_{n} .
$$

$\mathbf{2}^{\mathbf{0}} \mathbf{c}$. For any $j, k=1, \ldots, \mathfrak{m}_{n}, j \neq k$ introduce $\mathcal{P}_{j, k}=Q_{j}^{T} Q_{k}$. We have

$$
\begin{aligned}
b_{j, k}:= & \int_{\mathbb{R}^{2}} \frac{\mathbf{M}_{\varepsilon}\left(Q_{j}^{T} x\right) \mathbf{M}_{\varepsilon}\left(Q_{k}^{T} x\right)}{\mathbf{N}(x)} \mathrm{d} x=\int_{\mathbb{R}^{2}} \frac{\mathbf{M}_{\varepsilon}(x) \mathbf{M}_{\varepsilon}\left(\mathcal{P}_{j, k} x\right)}{\mathbf{N}(x)} \mathrm{d} x \\
= & \int_{\mathbb{R}^{2}} \frac{\lambda\left(x_{2} / \varepsilon\right) \mathbf{M}_{\varepsilon}\left(\mathcal{P}_{j, k} x\right)}{\mathbf{n}\left(x_{2}\right)} \mathrm{d} x+\int_{\mathbb{R}^{2}} \frac{\lambda\left(x_{1} / \varepsilon\right) \mathbf{M}_{\varepsilon}\left(\mathcal{P}_{j, k} x\right)}{\mathbf{n}\left(x_{1}\right)} \mathrm{d} x \\
& +\int_{\mathbb{R}^{2}} \frac{\Lambda(x) \mathbf{M}_{\varepsilon}\left(\mathcal{P}_{j, k} x\right)}{\mathbf{N}(x)} \mathrm{d} x .
\end{aligned}
$$

Taking into account that $\Lambda(x)=0$ for any $x \notin[-\varepsilon, \varepsilon]^{2}, \mathbf{N}(x) \geq 4^{-1} \mathbf{n}^{2}(0)$ on $[-\varepsilon, \varepsilon]^{2}$ and $M_{\varepsilon}$ is uniformly bounded, we obtain that for all $n$ large enough and some $C_{2}$ independent on $n$ and $L$

$$
\int_{\mathbb{R}^{2}} \frac{|\Lambda(x)|\left|\mathbf{M}_{\varepsilon}\left(\mathcal{P}_{j, k} x\right)\right|}{\mathbf{N}(x)} \mathrm{d} x \leq C_{2} L \varepsilon^{2} .
$$

Also, we have for sufficiently large $n$

$$
\begin{aligned}
& \int_{\mathbb{R}^{2}} \frac{\lambda\left(x_{2} / \varepsilon\right) \mathbf{M}_{\varepsilon}\left(\mathcal{P}_{j, k} x\right)}{\mathbf{n}\left(x_{2}\right)} \mathrm{d} x \leq 2 \mathbf{n}^{-1}(0)\left[\int_{\mathbb{R}^{2}} \lambda\left(x_{2} / \varepsilon\right) \mathbf{M}_{1}\left(\mathcal{P}_{j, k} x\right) \mathrm{d} x\right. \\
& \left.+\int_{\mathbb{R}^{2}} \lambda\left(x_{2} / \varepsilon\right) \mathbf{M}_{2}\left(\mathcal{P}_{j, k} x\right) \mathrm{d} x+L \varepsilon^{\beta} \int_{\mathbb{R}^{2}} \lambda\left(x_{2} / \varepsilon\right) \Lambda\left(\mathcal{P}_{j, k} x\right) \mathrm{d} x\right] .
\end{aligned}
$$

Putting for brevity $\boldsymbol{p}_{1}=p_{1}\left(Q_{j}, Q_{k}\right)$ and $\boldsymbol{p}_{2}=p_{2}\left(Q_{j}, Q_{k}\right)$ and making the change of variables: $\varepsilon z_{1}=\boldsymbol{p}_{1} x_{1}+\boldsymbol{p}_{2} x_{2}, \varepsilon z_{2}=x_{2}$ (first and third integrals), $\varepsilon z_{1}=\boldsymbol{p}_{2} x_{1}-\boldsymbol{p}_{1} z_{2}, \varepsilon z_{2}=x_{2}$ (second integral) we obtain since $\mathbf{n}$ and $\lambda$ are uniformly bounded for all $n$ large enough

$$
\left|\int_{\mathbb{R}^{2}} \frac{\lambda\left(x_{2} / \varepsilon\right) \mathbf{M}_{\varepsilon}\left(\mathcal{P}_{j, k} x\right)}{\mathbf{n}\left(x_{2}\right)} \mathrm{d} x\right| \leq\left[\left|\boldsymbol{p}_{1}\right| \wedge\left|\boldsymbol{p}_{2}\right|\right]^{-1}\left[C_{3} \varepsilon^{2}+C_{4} L \varepsilon^{2+\beta}\right]
$$

where $C_{3}$ and $C_{4}$ are the constants independent of $n$ and $L$.

imsart-generic ver. 2014/10/16 file: rotation-arXiv.tex date: February 26, 2020 
Since $\left|\boldsymbol{p}_{1}\right| \wedge\left|\boldsymbol{p}_{2}\right| \geq \delta_{n}$ in view of the definition of $\mathcal{Q}_{\delta_{n}}$ we obtain

$$
\left|\int_{\mathbb{R}^{2}} \frac{\lambda\left(x_{2} / \varepsilon\right) \mathbf{M}_{\varepsilon}\left(\mathcal{P}_{j, k} x\right)}{\mathbf{n}\left(x_{2}\right)} \mathrm{d} x\right| \leq C_{5} \varepsilon^{2} \delta_{n}^{-1}
$$

By the same computation we get

$$
\left|\int_{\mathbb{R}^{2}} \frac{\lambda\left(x_{1} / \varepsilon\right) \mathbf{M}_{\varepsilon}\left(\mathcal{P}_{j, k} x\right)}{\mathbf{n}\left(x_{1}\right)} \mathrm{d} x\right| \leq C_{5} \varepsilon^{2} \delta_{n}^{-1}
$$

Collecting the bounds obtained in (3.7), (3.8) and (3.9) we obtain

$$
\left|b_{j, k}\right| \leq C_{6} \varepsilon^{2} \delta_{n}^{-1} \leq C_{7} \varepsilon \mathfrak{n}^{-1}\left(\mathcal{Q}_{\delta_{n}}\right), \quad \forall j, k=1, \ldots, \mathfrak{m}_{n}, j \neq k .
$$

because $\delta_{n} \geq(\ln (n))^{\frac{2 \beta+2}{2 \beta+1}}\left(L^{-2} / n\right)^{\frac{1}{2 \beta+1}}$ in view of the assumption of the theorem and $\mathfrak{n}\left(\mathcal{Q}_{\delta_{n}}\right) \leq C_{8} \ln (n)$ in view of (2.2). It yields together with (3.5)

$$
\left|a_{j, k}\right|^{n} \leq e^{C_{9}}
$$

for any $j, k=1, \ldots, \mathfrak{m}_{n}, j \neq k$ and all $n$ large enough. This in its turn, together with (3.6) and (3.3) allows us to assert that (3.2) holds with $\Upsilon \leq$ $1+e^{C_{9}}$. The proof of the theorem is completed.

\subsection{Proofs of Theorems 3 and 4}

The proofs of Theorems 3 and 4 are essentially based on several auxiliary results. We starts with presenting such of them which will be used in the proofs of the both theorems simultaneously. Their proofs as well as the proofs of all auxiliary results are postponed to Appendix section. Set for any $f \in \mathcal{F}(\beta, L, \mathfrak{Q}), D \in \mathfrak{Q}$ and $x \in \mathbb{R}^{2}$

$$
\tau_{f}(D)=\left\{\begin{array}{cc}
\int_{\mathbb{R}^{2}} \boldsymbol{g}_{f}\left(\boldsymbol{p}_{1} \Gamma u\right) \boldsymbol{g}_{f}\left(\boldsymbol{p}_{1}^{-1} D \Omega x+\boldsymbol{p}_{2} \Omega \Gamma u\right) \mathrm{d} u, & D \neq \boldsymbol{Q}_{f} ; \\
f(x), & D=\boldsymbol{Q}_{f} .
\end{array}\right.
$$

where we have put $\boldsymbol{p}_{1}=p_{1}\left(D, \boldsymbol{Q}_{f}\right), \boldsymbol{p}_{2}=p_{2}\left(D, \boldsymbol{Q}_{f}\right)$. Set also

$$
\widetilde{f}_{h, d}(x)=n^{-1} \sum_{k=1}^{n} \mathcal{K}_{h}\left(d^{T}\left(X_{k}-x\right)\right), \widetilde{f}_{h, d_{\perp}}(x)=n^{-1} \sum_{k=1}^{n} \mathcal{K}_{h}\left(d_{\perp}^{T}\left(X_{k}-x\right)\right) .
$$

Lemma 1. For any $D \in \mathfrak{Q}, \beta>0, L>0, x \in \mathbb{R}^{2}, \mathcal{K} \in \mathbb{K}_{\beta}$ and $h>0$

$$
\sup _{f \in \mathcal{F}(\beta, L, \mathfrak{Q})}\left|\mathbb{E}_{f}\left[\widetilde{f}_{h, d}(x)\right] \mathbb{E}_{f}\left[\widetilde{f}_{h, d_{\perp}}(x)\right]-\tau_{f}(D)\right| \leq 2 C(\mathcal{K}, \beta, 1) L^{2} h^{\beta}
$$


Lemma 2. For any $\beta>0, L>0, x \in \mathbb{R}^{2}$ and $\mathcal{K} \in \mathbb{K}_{\beta}$

$$
\begin{gathered}
\sup _{D \in \mathfrak{Q}} \sup _{f \in \mathcal{F}(\beta, L, \mathfrak{Q})}\left|\mathbb{E}_{f}\left[\widetilde{f}_{h,\left(D, \boldsymbol{Q}_{f}\right)}(x)\right]-\mathbb{E}_{f}\left[\tilde{f}_{\eta, d}(x)\right] \mathbb{E}_{f}\left[\tilde{f}_{\eta, d_{\perp}}(x)\right]\right| \\
\leq 2 C(\mathcal{K}, \beta, \sqrt{2}) L^{2}\left(h^{\beta}+\eta^{\beta}\right), \quad \forall h, \eta>0 .
\end{gathered}
$$

Lemma 3. For any $D, Q \in \mathfrak{Q}$, and any $f \in \mathcal{F}(\beta, L, \mathfrak{Q})$

$$
\mathbb{E}_{f}\left[\widetilde{f}_{h,(D, Q)}(x)\right]=\mathbb{E}_{f}\left[\widetilde{f}_{h,(Q, D)}(x)\right] .
$$

This feature of the auxiliary estimator was called in Goldenshluger and Lepski (2012) the commutativity property.

Let $\mathfrak{I}_{n}$ be the set of all pairwise disjoint subsets of $\{1, \ldots, n\}$. For any $\mathcal{I} \in \mathfrak{I}_{n}$ its cardinality is denoted by $|\mathcal{I}|$ and $\widetilde{f}_{h, Q}^{(\mathcal{I})}$ will be used for the estimator built from $\left(X_{i}, i \in \mathcal{I}\right)$.

Proposition 1. Let $p \geq 1, \beta>0, L>0$, and $\mathcal{K} \in \mathbb{K}_{\beta}$ be fixed and set $\boldsymbol{h}=(\mu /|\mathcal{I}|)^{\frac{1}{2 \beta+1}}, \mu>0, \mathcal{I} \in \mathfrak{I}_{n}$. There exist $\mathbf{c}_{3}$ independent of $L$ such that

$$
\sup _{n \geq 1} \sup _{\mathcal{I} \in \mathfrak{I}_{n}} \sup _{\mu \in[1,|\mathcal{I}|]}(\mu /|\mathcal{I}|)^{-\frac{\beta}{2 \beta+1}} \sup _{\mathcal{F}(\beta, L, \mathfrak{Q})} \mathcal{R}_{n}^{(p)}\left[\widetilde{f}_{\boldsymbol{h}, \boldsymbol{Q}_{f}}^{(\mathcal{I})}, f\right] \leq \mathbf{c}_{3} L\left(L+L^{\frac{1}{p \vee 2}}\right) .
$$

\subsubsection{Proof of Theorem 3}

Let us formulate some auxiliary results the proofs of which are postponed to Appendix section.

Put $\mathbf{A}=12 \sqrt{10 p \alpha}(1+\sqrt{5 p})\left[1 \vee\|\mathcal{K}\|_{\infty}\right]+4 C(\mathcal{K}, \mathfrak{b}, \sqrt{2})$, where $\alpha=1 \vee \sup _{n \geq 3}\left\{\left[1 \vee \mathfrak{n}\left(\mathcal{Q}_{\delta_{n}}\right)\right] / \ln (n)\right\}$ is finite in view of $(2.2)$ and $(2.3)$.

Set for any $n \geq 3, \delta_{n}>0, \mathcal{Q}_{\delta_{n}} \in \mathbb{Q}_{\delta_{n}}$ and $f \in \mathcal{F}\left(\beta, L, \mathcal{Q}_{\delta_{n}}\right)$

$$
\begin{gathered}
\zeta_{n}(f, x)=\sup _{\substack{h \in \mathfrak{H}, D, Q \in \mathcal{Q}_{\delta_{n}}}}\left[\left|\widetilde{f}_{h,(D, Q)}(x)-\varkappa_{h}(D, Q, x)\right|-\mathfrak{a} \widehat{\mathcal{U}}_{n} \sqrt{\ln (n) / n h}\right]_{+} . \\
\varkappa_{h}(D, Q, x)= \begin{cases}\mathbb{E}_{f}\left[\widetilde{f}_{h,(D, Q)}(x)\right], & D \neq Q \\
\mathbb{E}_{f}\left[\widetilde{f}_{h, q}(x)\right] \mathbb{E}_{f}\left[\widetilde{f}_{h, q_{\perp}}(x)\right], & D=Q\end{cases}
\end{gathered}
$$

where $\mathfrak{a}=2^{-1} \mathbf{A}-2 C(\mathcal{K}, \mathfrak{b}, \sqrt{2})$.

Proposition 2. For any uniformly bounded kernel $\mathcal{K}$, an arbitrary sequence 
$\delta_{n}$ satisfying (2.3) and any $\mathcal{Q}_{\delta_{n}} \in \mathbb{Q}_{\delta_{n}}$ one has

$$
\begin{aligned}
& \limsup _{n \rightarrow \infty} n^{3 p} \sup _{\beta \in\left\{\beta_{1}, \beta_{2}\right\}} \sup _{f \in \mathcal{F}\left(\beta, L, \mathcal{Q}_{\delta_{n}}\right)} \mathbb{P}_{f}\left(\zeta_{n}(f, x) \neq 0\right)=0 ; \\
& \limsup _{n \rightarrow \infty} n^{p} \sup _{\beta \in\left\{\beta_{1}, \beta_{2}\right\}} \sup _{f \in \mathcal{F}\left(\beta, L, \mathcal{Q}_{\delta_{n}}\right)} \mathbb{E}_{f}\left[\zeta_{n}^{p}(f, x)\right]=0 ; \\
& \limsup _{n \rightarrow \infty} \sup _{\beta \in\left\{\beta_{1}, \beta_{2}\right\}} \sup _{f \in \mathcal{F}\left(\beta, L, \mathcal{Q}_{\delta_{n}}\right)} \mathbb{E}_{f}\left(\widehat{\mathcal{U}}_{n}^{p}\right) \leq \mathbf{C}_{p}(\mathcal{K}) L^{2 p}
\end{aligned}
$$

where $\mathbf{C}_{p}(\mathcal{K})>0$ is given in the proof of the proposition.

Proof of the theorem. We divide the proof into several steps.

$\mathbf{1}^{\mathbf{0}}$. For any $\beta \in(0, \mathfrak{b}]$ and $L>0$ set $\mathfrak{h}=\left(L^{-4} \ln (n) / n\right)^{\frac{1}{2 \beta+1}}$ and let $\mathcal{A}=\left\{R_{n}\left(\boldsymbol{Q}_{f}, \mathfrak{h}\right) \neq 0\right\}$. Our first goal is to prove the following result.

$$
\lim _{n \rightarrow \infty} \sup _{\beta \in\left\{\beta_{1}, \beta_{2}\right\}}\left(L^{\frac{2}{\beta}} \ln (n) / n\right)^{-\frac{p \beta}{2 \beta+1}} \sup _{\mathcal{F}\left(\beta, L, \mathcal{Q}_{\delta_{n}}\right)} \mathbb{E}_{f}|\widehat{f}-f(x)|^{p} 1_{\mathcal{A}}=0 .
$$

Note that for any $n \geq 1$ since $\widehat{h} \in \mathcal{H}$

$$
\left|\widetilde{f}_{\widehat{h}, \widehat{Q}}(x)\right| \leq\|\mathcal{K}\|_{\infty}^{2} \widehat{h}^{-2} \leq\|\mathcal{K}\|_{\infty}^{2} n^{2} .
$$

Hence (3.11) will be proved if we show that

$$
\limsup _{n \rightarrow \infty} \sup _{\beta \in\left\{\beta_{1}, \beta_{2}\right\}} n^{3 p} \sup _{\mathcal{F}\left(\beta, L, \mathcal{Q}_{\delta_{n}}\right)} \mathbb{P}_{f}(\mathcal{A})=0 .
$$

For any $\eta, \eta^{\prime} \in \mathfrak{H}, \eta^{\prime} \leq \eta \leq \mathfrak{h}$ we have in view of the definition of $\varkappa .(\cdot, \cdot, x)$

$$
\begin{aligned}
& \sup _{D \in \mathcal{Q}_{\delta_{n}}}\left|\widetilde{f}_{\eta,\left(D, \boldsymbol{Q}_{f}\right)}(x)-\widetilde{f}_{\eta^{\prime}, D}(x)\right| \leq \sup _{D \in \mathcal{Q}_{\delta_{n}}}\left|\widetilde{f}_{\eta,\left(D, \boldsymbol{Q}_{f}\right)}(x)-\varkappa_{\eta}\left(D, \boldsymbol{Q}_{f}, x\right)\right| \\
& +\sup _{D \in \mathcal{Q}_{\delta_{n}}}\left|\widetilde{f}_{\eta^{\prime}, D}(x)-\varkappa_{\eta^{\prime}}(D, D, x)\right|+\sup _{D \in \mathcal{Q}_{\delta_{n}}}\left|\varkappa_{\eta}\left(D, \boldsymbol{Q}_{f}, x\right)-\varkappa_{\eta^{\prime}}(D, D, x)\right| \\
& \leq 2 \mathfrak{a} \widehat{\mathcal{U}}_{n}\left(\ln (n) / n \eta^{\prime}\right)^{1 / 2}+2 \zeta_{n}(f, x) \\
& +\sup _{D \in \mathcal{Q}_{\delta_{n}}}\left|\mathbb{E}_{f}\left[\widetilde{f}_{\eta,\left(D, Q_{f}\right)}(x)\right]-\mathbb{E}_{f}\left[\widetilde{f}_{\eta^{\prime}, d}(x)\right] \mathbb{E}_{f}\left[\widetilde{f}_{\eta^{\prime}, d_{\perp}}(x)\right]\right|
\end{aligned}
$$

Taking into account that $(\ln (n) / n \mathfrak{h})^{1 / 2}=L^{2} \mathfrak{h}^{\beta}, \widehat{\mathcal{U}}_{n} \geq 1$ and applying Lemma 2 we get in view of the definition of $\mathbf{A}$ for any $\eta^{\prime} \leq \eta \leq \mathfrak{h}$

$$
\begin{gathered}
\sup _{D \in \mathcal{Q}_{\delta_{n}}}\left|\widetilde{f}_{\eta,\left(D, \boldsymbol{Q}_{f}\right)}(x)-\widetilde{f}_{\eta^{\prime}, D}(x)\right| \leq 2 \mathfrak{a} \widehat{\mathcal{U}}_{n}\left(\ln (n) / n \eta^{\prime}\right)^{1 / 2}+2 \zeta_{n}(f, x) \\
\left.+2 L^{2} C(K, \mathfrak{b}, \sqrt{2})\right]\left(\eta^{\beta}+\left(\eta^{\prime}\right)^{\beta}\right) \leq \widehat{\mathcal{U}}_{n}[2 \mathfrak{a}+4 C(\mathcal{K}, \mathfrak{b}, \sqrt{2})]\left(\ln (n) / n \eta^{\prime}\right)^{1 / 2} \\
+2 \zeta_{n}(f, x)=\mathbf{A} \widehat{\mathcal{U}}_{n}\left(\ln (n) / n \eta^{\prime}\right)^{1 / 2}+2 \zeta_{n}(f, x) .
\end{gathered}
$$

imsart-generic ver. 2014/10/16 file: rotation-arXiv.tex date: February 26, 2020 
Thus we have

$$
\sup _{\substack{\eta, \eta^{\prime} \in \mathfrak{S} ; \\ \eta^{\prime} \leq \eta \leq \mathfrak{h}}} \sup _{D \in \mathcal{Q}_{\delta}}\left[\left|\widetilde{f}_{\eta,\left(D, \boldsymbol{Q}_{f}\right)}(x)-\widetilde{f}_{\eta^{\prime}, D}(x)\right|-\mathbf{A} \widehat{\mathcal{U}}_{n}\left(\ln (n) / n \eta^{\prime}\right)^{1 / 2}\right]_{+} \leq 2 \zeta_{n} .
$$

The latter means that

$$
\mathbb{P}_{f}(\mathcal{A}) \leq \mathbb{P}_{f}\left(\zeta_{n}(f, x) \neq 0\right)
$$

and (3.12) follows from the first assertion of Proposition 2.

$\mathbf{2}^{\mathbf{0}}$. Denote $\overline{\mathcal{A}}$ the event complimentary to $\mathcal{A}$. Note that if $\overline{\mathcal{A}}$ is realized

$$
\begin{aligned}
R_{n}(\widehat{Q}, \widehat{h}) & \leq R_{n}(\widehat{Q}, \widehat{h})+\mathbf{A} \widehat{\mathcal{U}}_{n} \sqrt{\ln (n) / n \widehat{h}} \\
& \leq R_{n}\left(\boldsymbol{Q}_{f}, \mathfrak{h}\right)+\mathbf{A} \widehat{\mathcal{U}}_{n} \sqrt{\ln (n) / n \mathfrak{h}}=\mathbf{A} \widehat{\mathcal{U}}_{n} \sqrt{\ln (n) / n \mathfrak{h}}
\end{aligned}
$$

To get the second inequality we have used the definition of $(\widehat{h}, \widehat{Q})$.

Now let us prove the following inclusion.

$$
\overline{\mathcal{A}} \subseteq\{\widehat{h} \geq \mathfrak{h}\},
$$

Indeed, if $\overline{\mathcal{A}}$ is realized then

$$
\begin{aligned}
\mathbf{A} \widehat{\mathcal{U}}_{n} \sqrt{\ln (n) / n \widehat{h}} & \leq R_{n}(\widehat{Q}, \widehat{h})+\mathbf{A} \widehat{\mathcal{U}}_{n} \sqrt{\ln (n) / n \widehat{h}} \\
& \leq R_{n}\left(\boldsymbol{Q}_{f}, \mathfrak{h}\right)+\mathbf{A} \widehat{\mathcal{U}}_{n} \sqrt{\ln (n) / n \mathfrak{h}}=\mathbf{A} \widehat{\mathcal{U}}_{n} \sqrt{\ln (n) / n \mathfrak{h}}
\end{aligned}
$$

and (3.14) follows.

$\mathbf{3}^{\mathbf{0}}$. If $\overline{\mathcal{A}}$ is realized and, therefore $\widehat{h} \geq \mathfrak{h}$ in view of (3.14), we have

$$
\left|\widetilde{f}_{\widehat{h}, \widehat{Q}}(x)-\widetilde{f}_{\mathfrak{h}, \widehat{Q}}(x)\right| \leq R_{n}(\widehat{Q}, \widehat{h})+\mathbf{A} \widehat{\mathcal{U}}_{n} \sqrt{\ln (n) / n \mathfrak{h}} .
$$

This yields together with (3.13)

$$
\left|\widetilde{f}_{\widehat{h}, \widehat{Q}}(x)-\widetilde{f}_{\mathfrak{h}, \widehat{Q}}(x)\right| 1_{\overline{\mathcal{A}}} \leq 2 \mathbf{A} \widehat{\mathcal{U}}_{n} \sqrt{\ln (n) / n \mathfrak{h}} .
$$

Putting $\mathcal{B}=\left\{\widehat{Q}=\boldsymbol{Q}_{f}\right\}$ we deduce from (3.15)

$$
\left|\widetilde{f}_{\widehat{h}, \widehat{Q}}(x)-f(x)\right| 1_{\overline{\mathcal{A} \cap \mathcal{B}}} \leq 2 \mathbf{A} \widehat{\mathcal{U}}_{n} \sqrt{\ln (n) / n \mathfrak{h}}+\left|\widetilde{f}_{\mathfrak{h}, \boldsymbol{Q}_{f}}(x)-f(x)\right|(.3
$$

Also we obtain using (3.13)

$$
\begin{aligned}
\left|\widetilde{f}_{\mathfrak{h},\left(\boldsymbol{Q}_{f}, \widehat{Q}\right)}(x)-\widetilde{f}_{\mathfrak{h}, \boldsymbol{Q}_{f}}(x)\right| 1_{\overline{\mathcal{A}} \cap \overline{\mathcal{B}}} & \leq R_{n}(\widehat{Q}, \widehat{h})+\mathbf{A} \widehat{\mathcal{U}}_{n} \sqrt{\ln (n) / n \mathfrak{h}} \\
& \leq 2 \mathbf{A} \widehat{\mathcal{U}}_{n} \sqrt{\ln (n) / n \mathfrak{h}} \\
\left|\tilde{f}_{\mathfrak{h},\left(\widehat{Q}_{,}, \boldsymbol{Q}_{f}\right)}(x)-\widetilde{f}_{\mathfrak{h}, \widehat{Q}}(x)\right| 1_{\overline{\mathcal{A}} \cap \overline{\mathcal{B}}} & \leq R_{n}\left(\boldsymbol{Q}_{f}, \mathfrak{h}\right)+\mathbf{A} \widehat{\mathcal{U}}_{n} \sqrt{\ln (n) / n \mathfrak{h}}(3.1 \\
& =\mathbf{A} \widehat{\mathcal{U}}_{n} \sqrt{\ln (n) / n \mathfrak{h}}
\end{aligned}
$$

imsart-generic ver. 2014/10/16 file: rotation-arXiv.tex date: February 26, 2020 
We have in view of Lemma 3

$$
\begin{aligned}
& \mid \widetilde{f}_{\mathfrak{h},\left(\boldsymbol{Q}_{f}, \widehat{Q}\right)}(x)-\widetilde{f}_{\mathfrak{h},\left(\widehat{Q}, \boldsymbol{Q}_{f}\right)}(x)\left|\leq \sup _{D, Q \in \mathcal{Q}_{\delta_{n}}}\right| \widetilde{f}_{\mathfrak{h},(Q, D)}(x)-\widetilde{f}_{\mathfrak{h},(D, Q)}(x) \mid \\
& \leq \sup _{D, Q \in \mathcal{Q}_{\delta_{n}}}\left|\mathbb{E}_{f}\left[\widetilde{f}_{\mathfrak{h},(Q, D)}(x)\right]-\mathbb{E}_{f}\left[\widetilde{f}_{\mathfrak{h},(D, Q)}(x)\right]\right| \\
&+2 \mathbf{A} \widehat{\mathcal{U}}_{n} \sqrt{\ln (n) / n \mathfrak{h}}+2 \zeta_{n}(f, x)=2 \mathbf{A} \widehat{\mathcal{U}}_{n} \sqrt{\ln (n) / n \mathfrak{h}}+2 \zeta_{n}(f, x) .
\end{aligned}
$$

$\mathbf{4}^{\mathbf{0}}$. We obtain from (3.15), (3.17), (3.18) and (3.19)

$$
\left|\widetilde{f}_{\widehat{h}, \widehat{Q}}(x)-f(x)\right| 1_{\overline{\mathcal{A}} \cap \overline{\mathcal{B}}} \leq 7 \mathbf{A} \widehat{\mathcal{U}}_{n} \sqrt{\ln (n) / n \mathfrak{h}}+2 \zeta_{n}(f, x)+\left|\widetilde{f}_{\mathfrak{h}, \boldsymbol{Q}_{f}}(x)-f(x)\right| .
$$

It yields together with (3.16)

$$
\left|\widetilde{f}_{\widehat{h}, \widehat{Q}}(x)-f(x)\right| 1_{\overline{\mathcal{A}}} \leq 7 \mathbf{A} \widehat{\mathcal{U}}_{n} \sqrt{\ln (n) / n \mathfrak{h}}+2 \zeta_{n}(f, x)+\left|\widetilde{f}_{\mathfrak{h}, Q_{f}}(x)-f(x)\right| .
$$

Since $\ln (n) / n \mathfrak{h}=\left(L^{\frac{2}{\beta}} \ln (n) / n\right)^{\frac{2 \beta}{2 \beta+1}}$, we deduce from the second and third assertions of Proposition 2, (3.11) and (3.20)

$$
\begin{aligned}
\quad \limsup _{n \rightarrow \infty} \sup _{\beta \in\left\{\beta_{1}, \beta_{2}\right\}}\left(L^{\frac{1}{\beta}} \ln (n) / n\right)^{-\frac{\beta}{2 \beta+1}} \sup _{\mathcal{F}\left(\beta, L, \mathcal{Q}_{\delta_{n}}\right)} \mathcal{R}_{n}^{(p)}[\widehat{f}, f] \leq C_{p}\left\{L^{2}\right. \\
\left.+\limsup _{n \rightarrow \infty} \sup _{\beta \in\left\{\beta_{1}, \beta_{2}\right\}}\left(L^{\frac{1}{\beta}} \ln (n) / n\right)^{-\frac{\beta}{2 \beta+1}} \sup _{\mathcal{F}\left(\beta, L, \mathcal{Q}_{\delta_{n}}\right)} \mathcal{R}_{n}^{(p)}\left[\widetilde{f}_{\mathfrak{h}, \boldsymbol{Q}_{f}}(x), f\right]\right\}, \quad,
\end{aligned}
$$

where $C_{p}$ depends on $p$ and $\mathcal{K}$ only.

The assertion of the theorem follows now from (3.21) and Proposition 1 where one should choose $\mu=\min _{\beta \in\left\{\beta_{1}, \beta_{2}\right\}} L^{2 / \beta} \ln (n)$ and $\mathcal{I}=\{1, \ldots, n\}$.

\subsubsection{Proof of Theorem 4}

The proof of the theorem is similar to those of Theorem 3 and essentially based on the result formulated in Proposition 3 below.

$$
\begin{gathered}
\text { Put } \mathbf{B}=527730 p^{2} \sqrt{6}\left(\|\mathcal{K}\|_{1}^{2} \vee\|\mathcal{K}\|_{2}^{2} \vee\|\mathcal{K}\|_{\infty}^{2}\right)[9+4 \alpha]^{\frac{3 \beta+3}{2 \beta+1}}[C(\beta)]^{\frac{3}{2}} L^{\frac{4 \beta+8}{2 \beta+1}}+ \\
8 C(\mathcal{K}, \mathfrak{b}, \sqrt{2}) L^{2}, \text { where } C(\beta):=1 \vee \sup _{n \geq 3}\left\{\left[\ln ^{2}(n) / n\right]^{\frac{2 \beta}{2 \beta+1}}[\ln (n)]^{\frac{2}{2 \beta+1}}\right\} .
\end{gathered}
$$

Set for any $n \geq 3, \delta_{n}>0, \mathcal{Q}_{\delta_{n}} \in \mathbb{Q}_{\delta_{n}}$ and $f \in \mathcal{F}\left(\beta, L, \mathcal{Q}_{\delta_{n}}\right)$

$$
\chi_{\mathbf{i}}(f, x)=\sup _{D, Q \in \mathcal{Q}_{\delta_{n}}}\left[\left|\widetilde{f}_{h_{\mathbf{i}},(D, Q)}^{(\mathbf{i})}(x)-\varkappa_{h_{\mathbf{i}}}(D, Q, x)\right|-\mathbf{C} L^{2} h_{\mathbf{i}}^{\beta}\right]_{+},
$$

where $\varkappa_{h}(\cdot, \cdot, x), h>0$, is defined in $(3.10)$ and $\mathbf{C}=2^{-1} \mathbf{B}-4 C(\mathcal{K}, \beta, \sqrt{2}) L^{2}$. 
Proposition 3. For any $\beta>0, L>0, \mathcal{K} \in \mathbb{K}_{\beta}$, an arbitrary sequence $\delta_{n}$ satisfying (2.3) with $\mathfrak{b}=\beta, \mathcal{Q}_{\delta_{n}} \in \mathbb{Q}_{\delta_{n}}$ and any $\mathbf{i}=1, \ldots, \mathbf{i}^{*}$ one has

$$
\begin{aligned}
& \sup _{n \geq 3} \sup _{\mathbf{i}=1, \ldots, \mathbf{i}^{*}}\left(\frac{\omega_{\mathbf{i}-1} \mathbf{n}_{\mathbf{i}}}{\mathbf{n}_{\mathbf{i}-1} \omega_{\mathbf{i}}}\right)^{\frac{p \beta}{2 \beta+1}} \sup _{f \in \mathcal{F}\left(\beta, L, \mathcal{Q}_{\delta_{n}}\right)} \mathbb{P}_{f}\left\{\chi_{\mathbf{i}}(f, x) \neq 0\right\}=: \mathbf{P}<1 ; \\
& \sup _{n \geq 3 \mathbf{i}=1, \ldots, \mathbf{i}^{*}}\left(L^{\frac{2}{\beta}} \omega_{\mathbf{i}} / \mathbf{n}_{\mathbf{i}}\right)^{-\frac{p \beta}{2 \beta+1}} \sup _{f \in \mathcal{F}\left(\beta, L, \mathcal{Q}_{\delta_{n}}\right)} \mathbb{E}_{f}\left\{\chi_{\mathbf{i}}^{p}(f, x)\right\}=: \mathbf{E}<\infty .
\end{aligned}
$$

Proof of the theorem. Throughout the proof we will understand $\varkappa_{h_{\mathbf{i}}}(\cdot, \cdot, x)$ introduced in (3.10) as the mapping defined on $\mathcal{Q}_{\delta_{n}} \times \mathcal{Q}_{\delta_{n}}$ (its explicit expression via some integral operators can be easily obtained). It allows us to introduce below random variables $\varkappa_{h_{\mathbf{i}}}\left(\widehat{Q}^{(\mathbf{i})}, \cdot, x\right), \mathbf{i}=1, \ldots, \mathbf{i}^{*}$.

$\mathbf{1}^{\mathbf{0}}$. Introduce the random event $\mathcal{Z}^{(\mathbf{i})}=\left\{R_{n}^{(\mathbf{i})}\left(\widehat{Q}^{(\mathbf{i})}\right)=0\right\}$. If $\mathcal{Z}^{(\mathbf{i})}$ is realized, we assert, using the definitions of $\widehat{Q}^{(\mathbf{i})}$ and $\breve{f}^{(\mathbf{i})}$ that

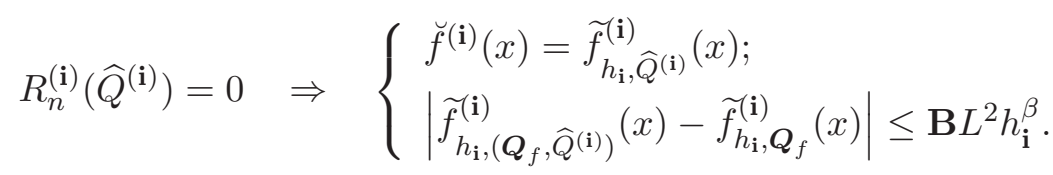

Note that

$$
\begin{gathered}
\left|\widetilde{f}_{h_{\mathbf{i}}, \widehat{Q}^{(\mathbf{i})}}^{(\mathbf{i})}(x)-f(x)\right| \leq\left|\widetilde{f}_{h_{\mathbf{i}}, \widehat{Q}^{(\mathbf{i})}}^{(i)}(x)-\varkappa_{h_{\mathbf{i}}}\left(\widehat{Q}^{(\mathbf{i})}, \widehat{Q}^{(\mathbf{i})}, x\right)\right| \\
+\left|\varkappa_{h_{\mathbf{i}}}\left(\widehat{Q}^{(\mathbf{i})}, \widehat{Q}^{(\mathbf{i})}, x\right)-f(x)\right| \leq \mathbf{C} L^{2} h_{\mathbf{i}}^{\beta}+\chi_{\mathbf{i}}(f, x) \\
+\sup _{Q \in \mathcal{Q}_{\delta_{n}}}\left|\varkappa_{h_{\mathbf{i}}}(Q, Q, x)-\varkappa_{h_{\mathbf{i}}}\left(Q, \boldsymbol{Q}_{f}, x\right)\right|+\left|\varkappa_{h_{\mathbf{i}}}\left(\widehat{Q}^{(\mathbf{i})}, \boldsymbol{Q}_{f}, x\right)-f(x)\right| \\
\leq \mathbf{C} L^{2} h_{\mathbf{i}}^{\beta}+\chi_{\mathbf{i}}(f, x)+4 C(\mathcal{K}, \beta, \sqrt{2}) L^{2} h_{\mathbf{i}}^{\beta}+\left|\varkappa_{h_{\mathbf{i}}}\left(\widehat{Q}^{(\mathbf{i})}, \boldsymbol{Q}_{f}, x\right)-f(x)\right| \\
\leq 2^{-1} \mathbf{B} L^{2} h_{\mathbf{i}}^{\beta}+\chi_{\mathbf{i}}(f, x)+\left|\varkappa_{h_{\mathbf{i}}}\left(\widehat{Q}^{(\mathbf{i})}, \boldsymbol{Q}_{f}, x\right)-f(x)\right| .
\end{gathered}
$$

To get the penultimate inequality we have used Lemma 2 while the last one follows from the definition of C. Also in view of Lemma 3 for all $D \in \mathcal{Q}_{\delta_{n}}$

$$
\begin{gathered}
\varkappa_{h_{\mathbf{i}}}\left(\widehat{Q}^{(\mathbf{i})}, D, x\right)=\sum_{Q \in \mathcal{Q}_{\delta_{n}}} \varkappa_{h_{\mathbf{i}}}(Q, D, x) 1_{\widehat{Q}^{(\mathbf{i})}=Q} \\
=\sum_{\substack{Q \in \mathcal{Q}_{\delta_{n}} \\
Q \neq D}} \mathbb{E}_{f}\left[\widetilde{f}_{h_{\mathbf{i}},(Q, D)}^{(\mathbf{i})}(x)\right] 1_{\widehat{Q}^{(\mathbf{i})}=Q}+\varkappa_{h_{\mathbf{i}}}(D, D, x) 1_{\widehat{Q}^{(\mathbf{i})}=D} \\
=\sum_{\substack{Q \in \mathcal{Q}_{\delta_{n}} \\
Q \neq D}} \mathbb{E}_{f}\left[\widetilde{f}_{h_{\mathbf{i}},(D, Q)}^{(\mathbf{i})}(x)\right] 1_{\widehat{Q}^{(\mathbf{i})}=Q}+\varkappa_{h_{\mathbf{i}}}(D, D, x) 1_{\widehat{Q}^{(\mathbf{i})}=D}=\varkappa_{h_{\mathbf{i}}}\left(D, \widehat{Q}^{(\mathbf{i})}, x\right) .
\end{gathered}
$$


Thus, if $\mathcal{Z}^{(\mathbf{i})}$ is realized we have in view of (3.24)

$$
\begin{aligned}
& \left|\varkappa_{h_{\mathbf{i}}}\left(\widehat{Q}^{(\mathbf{i})}, \boldsymbol{Q}_{f}, x\right)-f(x)\right|=\left|\varkappa_{h_{\mathbf{i}}}\left(\boldsymbol{Q}_{f}, \widehat{Q}^{(\mathbf{i})}, x\right)-f(x)\right| \\
& \leq\left|\varkappa_{h_{\mathbf{i}}}\left(\boldsymbol{Q}_{f}, \widehat{Q}^{(\mathbf{i})}, x\right)-\widetilde{f}_{h_{\mathbf{i}},\left(\boldsymbol{Q}_{f}, \widehat{Q}^{(\mathbf{i})}\right)}^{(\mathbf{i})}(x)\right|+\left|\widetilde{f}_{h_{\mathbf{i}},\left(\boldsymbol{Q}_{f}, \widehat{Q}^{(\mathbf{i})}\right)}^{(\mathbf{i})}(x)-\widetilde{f}_{h_{\mathbf{i}}, \boldsymbol{Q}_{f}}^{(\mathbf{i})}(x)\right| \\
& +\left|\widetilde{f}_{h_{\mathbf{i}}, \boldsymbol{Q}_{f}}^{(\mathbf{i})}(x)-f(x)\right| \leq \mathbf{C} L^{2} h_{\mathbf{i}}^{\beta}+\chi_{\mathbf{i}}(f, x)+\mathbf{B} L^{2} h_{\mathbf{i}}^{\beta}+\left|\widetilde{f}_{h_{\mathbf{i}}, \boldsymbol{Q}_{f}}^{(\mathbf{i})}(x)-f(x)\right| .
\end{aligned}
$$

It yields together with $(3.23)$

$$
\left|\widetilde{f}_{h_{\mathbf{i}}, \widehat{Q}^{(\mathbf{i})}}^{(\mathbf{i})}(x)-f(x)\right| 1_{\mathcal{Z}^{(\mathbf{i})}} \leq 2 \mathbf{B} L^{2} h_{\mathbf{i}}^{\beta}+2 \chi_{\mathbf{i}}(f, x)+\left|\widetilde{f}_{h_{\mathbf{i}}, \boldsymbol{Q}_{f}}^{(\mathbf{i})}(x)-f(x)\right| .
$$

First, we deduce from Proposition 1

$$
\left.\sup _{n \geq 3} \sup _{\mathbf{i}=1, \ldots, \mathbf{i}^{*}}\left(L^{\frac{2}{\beta}} \omega_{\mathbf{i}} / \mathbf{n}_{\mathbf{i}}\right)^{-\frac{p \beta}{2 \beta+1}} \sup _{\mathcal{F}\left(\beta, L, \mathcal{Q}_{\delta_{n}}\right)}\left[\mathcal{R}_{n}^{(p)}\left[\widetilde{f}_{h_{\mathbf{i}}, \boldsymbol{Q}_{f}}^{(\mathbf{i})}, f\right]\right]^{p}\right\}=: C_{1}<\infty .
$$

Next, taking into account that $L^{2} h_{\mathbf{i}}^{\beta}=\left(L^{\frac{2}{\beta}} \omega_{\mathbf{i}} / \mathbf{n}_{\mathbf{i}}\right)^{\frac{\beta}{2 \beta+1}}$ and denoting

$$
\mathbf{R}=\sup _{n \geq 3} \sup _{\mathbf{i}=1, \ldots, \mathbf{i}^{*}}\left(L^{\frac{2}{\beta}} \omega_{\mathbf{i}} / \mathbf{n}_{\mathbf{i}}\right)^{-\frac{p \beta}{2 \beta+1}} \sup _{\mathcal{F}\left(\beta, L, \mathcal{Q}_{\delta_{n}}\right)} \mathbb{E}_{f}\left|\breve{f}^{(\mathbf{i})}(x)-f(x)\right|^{p} 1_{\mathcal{Z}^{(\mathbf{i})}}
$$

we deduce from (3.22), (3.25) and the second assertion of Proposition 3

$$
\mathbf{R} \leq 3^{p}\left\{(2 \mathbf{B})^{p}+2^{p} \mathbf{E}+C_{1}\right\} .
$$

$\mathbf{2}^{\mathbf{0}}$. In view of the definition of $\breve{f}^{(\mathbf{i})}$ we have

$$
\begin{aligned}
& \mathbb{E}_{f}\left|\breve{f}^{(\mathbf{i})}(x)-f(x)\right|^{p} 1_{\overline{\mathcal{Z}}^{(\mathbf{i})}}=\mathbb{E}_{f}\left\{\left|\breve{f}^{(\mathbf{i}-1)}(x)-f(x)\right|^{p} 1_{\overline{\mathcal{Z}}^{(\mathbf{i})}}\right\} \\
& =\mathbb{E}_{f}\left|\breve{f}^{(\mathbf{i}-1)}(x)-f(x)\right|^{p} \mathbb{P}_{f}\left(\overline{\mathcal{Z}}^{(\mathbf{i})}\right),
\end{aligned}
$$

since $X^{(\mathbf{i})}$ and $X^{(\mathbf{i}-1)}$ are the independent collections of random variables. Note that in view of the definition of $\varkappa_{h_{\mathbf{i}}}(\cdot, \cdot, x)$

$$
\begin{aligned}
& \sup _{D \in \mathcal{Q}_{\delta_{n}}}\left|\widetilde{f}_{h_{\mathbf{i}},\left(D, \boldsymbol{Q}_{f}\right)}^{(\mathbf{i})}(x)-\widetilde{f}_{h_{\mathbf{i}}, D}^{(\mathbf{i})}(x)\right| \leq \sup _{D \in \mathcal{Q}_{\delta_{n}}}\left|\widetilde{f}_{h_{\mathbf{i}},\left(D, \boldsymbol{Q}_{f}\right)}^{(\mathbf{i})}(x)-\varkappa_{h_{\mathbf{i}}}\left(D, \boldsymbol{Q}_{f}, x\right)\right| \\
& +\sup _{D \in \mathcal{Q}_{\delta_{n}}}\left|\widetilde{f}_{h_{\mathbf{i}}, D}^{(\mathbf{i})}(x)-\varkappa_{h_{\mathbf{i}}}(D, D, x)\right|+\sup _{D \in \mathcal{Q}_{\delta_{n}}}\left|\varkappa_{h_{\mathbf{i}}}\left(D, \boldsymbol{Q}_{f}, x\right)-\varkappa_{h_{\mathbf{i}}}(D, D, x)\right| \\
& \leq 2 \chi_{\mathbf{i}}(f, x)+2 \mathbf{C} L^{2} h_{\mathbf{i}}^{\beta}+\sup _{D \in \mathcal{Q}_{\delta_{n}}}\left|\varkappa_{h_{\mathbf{i}}}\left(D, \boldsymbol{Q}_{f}, x\right)-\varkappa_{h_{\mathbf{i}}}(D, D, x)\right| \\
& \leq 2 \chi_{\mathbf{i}}(f, x)+2 \mathbf{C} L^{2} h_{\mathbf{i}}^{\beta}+4 C L^{2}(\mathcal{K}, \beta, \sqrt{2}) h_{\mathbf{i}}^{\beta}=2 \chi_{\mathbf{i}}(f, x)+\mathbf{B} L^{2} h_{\mathbf{i}}^{\beta} .
\end{aligned}
$$


To get the second inequality we have used Lemma 2 while the last equality follows from the definition of $\mathbf{C}$. Noting that the definition of $\widehat{Q}^{(\mathbf{i})}$ implies the inclusion $\overline{\mathcal{Z}}^{(\mathbf{i})} \subseteq\left\{R_{n}^{(\mathbf{i})}\left(\boldsymbol{Q}_{f}\right) \neq 0\right\}$ we obtain

$$
\begin{aligned}
\overline{\mathcal{Z}}^{(\mathbf{i})} \subseteq\left\{R_{n}^{(\mathbf{i})}\left(\boldsymbol{Q}_{f}\right) \neq 0\right\} & =\left\{\sup _{D \in \mathcal{Q}_{\delta_{n}}}\left|\widetilde{f}_{h_{\mathbf{i}},\left(D, \boldsymbol{Q}_{f}\right)}^{(\mathbf{i})}(x)-\widetilde{f}_{h_{\mathbf{i}}, D}^{(\mathbf{i})}(x)\right|>\mathbf{B} L^{2} h_{\mathbf{i}}^{\beta}\right\} \\
& \subseteq\left\{\chi_{\mathbf{i}}(f, x) \neq 0\right\} .
\end{aligned}
$$

Denoting by $\omega_{0}=\ln (n), \mathbf{n}_{\mathbf{0}}=\lfloor n / 4\rfloor$ and

$$
\mathbf{e}_{\mathbf{i}}=\left(L^{\frac{2}{\beta}} \omega_{\mathbf{i}} / \mathbf{n}_{\mathbf{i}}\right)^{-\frac{p \beta}{2 \beta+1}} \sup _{\mathcal{F}\left(\beta, L, \mathcal{Q}_{\delta_{n}}\right)} \mathbb{E}_{f}|\breve{f}(\mathbf{i})(x)-f(x)|^{p} .
$$

we deduce from (3.27), (3.28) and the first assertion of Proposition 3

$$
\mathbf{e}_{\mathbf{i}} \leq \mathbf{R}+\mathbf{P e}_{\mathbf{i}-1}, \quad \forall \mathbf{i}=1, \ldots, \mathbf{i}^{*}, \forall n \geq 3 .
$$

It yields together with (3.26) since $\mathbf{P}<1$ for all $n \geq 3$

$$
\mathbf{e}_{\mathbf{i}^{*}} \leq \mathbf{P}^{\mathbf{i}^{*}} \mathbf{e}_{0}+\mathbf{R}(1-\mathbf{P})^{-1} \leq \mathbf{e}_{0}+3^{p}\left\{(2 \mathbf{B})^{p}+2^{p} \mathbf{E}+C_{1}\right\}(1-\mathbf{P})^{-1} .
$$

Since $\breve{f}^{(\mathbf{0})}(x)=\widehat{f}(x)$ we deduce from Theorem 3 that

$$
\limsup _{n \rightarrow \infty} \mathbf{e}_{0}<\infty
$$

that completes the proof of the theorem.

\section{Proofs of Lemmas 1-3 and Proposition 1}

The proofs of Lemmas 1-3 are based on the following result proved in the end of this section.

Lemma 4. For any $g \in \mathcal{G}(\beta, L)$ and any $2 \times 2$ matrix $\Psi=\left(\psi_{1}^{T}, \psi_{2}^{T}\right)$

$$
\sup _{y \in \mathbb{R}^{2}}\left|\int_{\mathbb{R}^{2}} K(t) g(y+\Psi t h) \mathrm{d} t-g(y)\right| \mathrm{d} \underline{u} \leq C\left(\mathcal{K}, \beta, \psi^{*}\right) L^{2} h^{\beta}, \quad \forall h>0,
$$

where $\psi^{*}=\left\|\psi_{1}\right\| \vee\left\|\psi_{2}\right\|$.

Proof of Lemma 1. $\mathbf{1}^{\mathbf{0}}$. We obviously have

$$
\begin{aligned}
\mathcal{E}_{h}(f, D, x) & :=\mathbb{E}_{f}\left[\widetilde{f}_{h, d}(x)\right] \mathbb{E}_{f}\left[\widetilde{f}_{h, d_{\perp}}(x)\right] \\
& =\left[\int_{\mathbb{R}^{2}} \mathcal{K}_{h}\left(d^{T}(u-x)\right) f(u) \mathrm{d} u\right]\left[\int_{\mathbb{R}^{2}} \mathcal{K}_{h}\left(d_{\perp}^{T}(u-x)\right) f(u) \mathrm{d} u\right] \\
& =: \mathcal{E}_{h}^{\prime}(f, D, x) \mathcal{E}_{h}^{\prime \prime}(f, D, x) .
\end{aligned}
$$

imsart-generic ver. 2014/10/16 file: rotation-arXiv.tex date: February 26, 2020 
Since $f(u)=\boldsymbol{g}_{f}\left(\boldsymbol{Q}_{f}^{T} u\right), u \in \mathbb{R}^{2}$, denoting for brevity $\boldsymbol{Q}_{f}=\left(\boldsymbol{q}, \boldsymbol{q}_{\perp}\right)$ and by $\boldsymbol{g}_{i}, i=1,2$, the marginals of $\boldsymbol{g}_{f}$, we get

$$
\begin{aligned}
& \mathcal{E}_{h}^{\prime}(f, D, x)=\int_{\mathbb{R}^{2}} \mathcal{K}_{h}\left(u_{1}\right) \boldsymbol{g}_{1}\left(\boldsymbol{q}^{T} x+\boldsymbol{q}^{T} d u_{1}+\boldsymbol{q}^{T} d_{\perp} u_{2}\right) \times \\
& \boldsymbol{g}_{2}\left(\boldsymbol{q}_{\perp}^{T} x+\boldsymbol{q}_{\perp}^{T} d u_{1}+\boldsymbol{q}_{\perp}^{T} d_{\perp} u_{2}\right) \mathrm{d} u_{1} \mathrm{~d} u_{2} \\
& =\int_{\mathbb{R}^{2}} \mathcal{K}\left(s_{1}\right) \boldsymbol{g}_{1}\left(\boldsymbol{q}^{T} x+\boldsymbol{p}_{2} s_{1} h+\boldsymbol{p}_{1} s_{3}\right) \boldsymbol{g}_{2}\left(q_{\perp}^{T} x-\boldsymbol{p}_{1} s_{1} h+\boldsymbol{p}_{2} s_{3}\right) \mathrm{d} s_{1} \mathrm{~d} s_{3}, \\
& \mathcal{E}_{h}^{\prime \prime}(f, D, x)=\int_{\mathbb{R}^{2}} \mathcal{K}_{h}\left(u_{2}\right) \boldsymbol{g}_{1}\left(\boldsymbol{q}^{T} x+\boldsymbol{q}^{T} d u_{1}+\boldsymbol{q}^{T} d_{\perp} u_{2}\right) \times \\
& \boldsymbol{g}_{2}\left(\boldsymbol{q}_{\perp}^{T} x+\boldsymbol{q}_{\perp}^{T} d u_{1}+\boldsymbol{q}_{\perp}^{T} d_{\perp} u_{2}\right) \mathrm{d} u_{1} \mathrm{~d} u_{2} \\
& =\int_{\mathbb{R}^{2}} \mathcal{K}\left(s_{2}\right) \boldsymbol{g}_{1}\left(\boldsymbol{q}^{T} x+\boldsymbol{p}_{2} s_{4}+\boldsymbol{p}_{1} s_{2} h\right) \boldsymbol{g}_{2}\left(\boldsymbol{q}_{\perp}^{T} x-\boldsymbol{p}_{1} s_{4}+\boldsymbol{p}_{2} s_{2} h\right) \mathrm{d} s_{2} \mathrm{~d} s_{4} .
\end{aligned}
$$

Thus we obtain that

$$
\begin{aligned}
& \mathcal{E}_{h}(f, D, x) \\
& =\int_{\mathbb{R}^{4}} K(\bar{s}) \boldsymbol{g}_{f}\left(\boldsymbol{Q}_{f}^{T} x+\boldsymbol{p}_{2} \bar{s} h+\boldsymbol{p}_{1} \Gamma \underline{s}\right) \boldsymbol{g}_{f}\left(\boldsymbol{Q}_{f}^{T} x+\boldsymbol{p}_{1} \Gamma \Omega \bar{s} h+\boldsymbol{p}_{2} \Omega \underline{s}\right) \mathrm{d} s .
\end{aligned}
$$

If $D=\boldsymbol{Q}_{f}$ that implies $\boldsymbol{p}_{1}=0$ and $\boldsymbol{p}_{2}=1$ we get

$$
\begin{aligned}
\mathcal{E}_{h}(f, D, x) & =\int_{\mathbb{R}^{4}} K(\bar{s}) \boldsymbol{g}_{f}\left(\boldsymbol{Q}_{f}^{T} x+\bar{s} h\right) \boldsymbol{g}_{f}\left(\boldsymbol{Q}_{f}^{T} x+\Omega \underline{s}\right) \mathrm{d} s \\
& =\int_{\mathbb{R}^{2}} K(\bar{s}) \boldsymbol{g}_{f}\left(\boldsymbol{Q}_{f}^{T} x+\bar{s} h\right) \mathrm{d} \bar{s},
\end{aligned}
$$

since $\boldsymbol{g}_{f}$ is a probability density. The assertion of the lemma in this case follows from Lemma 4 . If $D \neq \boldsymbol{Q}_{f}\left(\boldsymbol{p}_{1} \neq 0\right)$, making the change of variables $\boldsymbol{Q}_{f}^{T} x+\boldsymbol{p}_{1} \Gamma \underline{s}=\boldsymbol{p}_{1} \Gamma \underline{t}$ and noting that $\Gamma^{-1}=\Gamma$ we come to

$$
\begin{aligned}
& \mathcal{E}_{h}(f, D, x) \\
& =\int_{\mathbb{R}^{4}} K(\bar{t}) \boldsymbol{g}_{f}\left(\boldsymbol{p}_{2} \bar{t} h+\boldsymbol{p}_{1} \Gamma \underline{t}\right) \boldsymbol{g}_{f}\left(\left[I-\boldsymbol{p}_{2} \boldsymbol{p}_{1}^{-1} \Omega \Gamma\right] \boldsymbol{Q}_{f}^{T} x+\boldsymbol{p}_{1} \Gamma \Omega \bar{t} h+\boldsymbol{p}_{2} \Omega \underline{t}\right) \mathrm{d} t .
\end{aligned}
$$

Noting that $I-\boldsymbol{p}_{2} \boldsymbol{p}_{1}^{-1} \Omega \Gamma=\boldsymbol{p}_{1}^{-1} D^{T} \boldsymbol{Q}_{f} \Gamma \Omega$, we get

$$
\left[I-\boldsymbol{p}_{2} \boldsymbol{p}_{1}^{-1} \Omega \Gamma\right] \boldsymbol{Q}_{f}^{T} x=\boldsymbol{p}_{1}^{-1}\left(\begin{array}{c}
d_{\perp}^{T} x \\
-d^{T} x
\end{array}\right)
$$

Thus we have 


$$
\begin{aligned}
& \boldsymbol{g}_{f}\left(\left[I-\boldsymbol{p}_{2} \boldsymbol{p}_{1}^{-1} \Omega \Gamma\right] \boldsymbol{Q}_{f}^{T} x+\boldsymbol{p}_{1} \Gamma \Omega \bar{t} h+\boldsymbol{p}_{2} \Omega \underline{t}\right) \\
& =\boldsymbol{g}_{1}\left(\boldsymbol{p}_{1}^{-1} d_{\perp}^{T} x+\boldsymbol{p}_{1} t_{2} h+\boldsymbol{p}_{2} t_{4}\right) \boldsymbol{g}_{2}\left(-\boldsymbol{p}_{1}^{-1} d^{T} x-\boldsymbol{p}_{1} t_{1} h+\boldsymbol{p}_{2} t_{3}\right)
\end{aligned}
$$

and, since $\boldsymbol{g}_{2}$ is symmetric

$$
\begin{aligned}
& \boldsymbol{g}_{f}\left(\left[I-\boldsymbol{p}_{2} \boldsymbol{p}_{1}^{-1} \Omega \Gamma\right] \boldsymbol{Q}_{f}^{T} x+\boldsymbol{p}_{1} \Gamma \Omega \bar{t} h+\boldsymbol{p}_{2} \Omega \underline{t}\right) \\
& =\boldsymbol{g}_{1}\left(\boldsymbol{p}_{1}^{-1} d_{\perp}^{T} x+\boldsymbol{p}_{1} t_{2} h+\boldsymbol{p}_{2} t_{4}\right) \boldsymbol{g}_{2}\left(\boldsymbol{p}_{1}^{-1} d^{T} x+\boldsymbol{p}_{1} t_{1} h-\boldsymbol{p}_{2} t_{3}\right)
\end{aligned}
$$

Noting that $\left(d_{\perp} d\right)^{T}=D \Omega$, we obtain finally

$$
\begin{aligned}
& \mathcal{E}_{h}(f, D, x) \\
& =\int_{\mathbb{R}^{4}} K(\bar{t}) \boldsymbol{g}_{f}\left(\boldsymbol{p}_{2} \bar{t} h+\boldsymbol{p}_{1} \Gamma \underline{t}\right) \boldsymbol{g}_{f}\left(\boldsymbol{p}_{1}^{-1} D \Omega x+\boldsymbol{p}_{1} \Omega \bar{t} h+\boldsymbol{p}_{2} \Omega \Gamma \underline{t}\right) \mathrm{d} t .(4
\end{aligned}
$$

Consider now two cases.

$\mathbf{2}^{\mathbf{0}} \mathbf{a}$. If $\left|\boldsymbol{p}_{2}\right| \geq\left|\boldsymbol{p}_{1}\right|$ using $\Omega D \Omega=D, \Omega^{2}=I, \Gamma^{2}=I$ and making the change of variables $\bar{t}=\bar{v}$,

$$
\boldsymbol{p}_{1}^{-1} D \Omega x+\boldsymbol{p}_{1} \Omega \bar{t} h+\boldsymbol{p}_{2} \Omega \Gamma \underline{t}=\underline{v} \Rightarrow \underline{t}=\boldsymbol{p}_{2}^{-1} \Gamma \Omega \underline{v}-\boldsymbol{p}_{2}^{-1}\left[\boldsymbol{p}_{1}^{-1} \Gamma D x+\boldsymbol{p}_{1} \Gamma \bar{v} h\right]
$$

we obtain (remind that $\boldsymbol{g}_{f}$ is a symmetric function)

$$
\begin{aligned}
& \mathcal{E}_{h}(f, D, x) \\
& =\boldsymbol{p}_{2}^{-2} \int_{\mathbb{R}^{4}} \boldsymbol{g}_{f}(\underline{v}) K(\bar{v}) \boldsymbol{g}_{f}\left(\boldsymbol{p}_{2}^{-1} D x-\left[\boldsymbol{p}_{2}-\boldsymbol{p}_{1}^{2} \boldsymbol{p}_{2}^{-1}\right] \bar{v} h-\boldsymbol{p}_{1} \boldsymbol{p}_{2}^{-1} \Omega \underline{v}\right) \mathrm{d} v .
\end{aligned}
$$

Hence, taking into account that $\boldsymbol{g}_{f}$ is a probability density we deduce from Lemma 4 that

$$
\begin{aligned}
& \quad\left|\mathcal{E}_{h}(f, D, x)-\boldsymbol{p}_{2}^{-2} \int_{\mathbb{R}^{2}} \boldsymbol{g}_{f}(\underline{v}) \boldsymbol{g}_{f}\left(\boldsymbol{p}_{2}^{-1} D x-\boldsymbol{p}_{1} \boldsymbol{p}_{2}^{-1} \Omega \underline{v}\right) \mathrm{d} \underline{v}\right| \\
& =\boldsymbol{p}_{2}^{-2} \mid \int_{\mathbb{R}^{2}} \boldsymbol{g}_{f}(\underline{v})\left[\int_{\mathbb{R}^{2}} K(\bar{v}) \boldsymbol{g}_{f}\left(\boldsymbol{p}_{2}^{-1} D x-\left[\boldsymbol{p}_{2}-\boldsymbol{p}_{1}^{2} \boldsymbol{p}_{2}^{-1}\right] \bar{v} h-\boldsymbol{p}_{1} \boldsymbol{p}_{2}^{-1} \Omega \underline{v}\right) \mathrm{d} \bar{v}\right. \\
& \left.\quad-\int_{\mathbb{R}^{2}} \boldsymbol{g}_{f}\left(\boldsymbol{p}_{2}^{-1} D x-\boldsymbol{p}_{1} \boldsymbol{p}_{2}^{-1} \Omega \underline{v}\right)\right] \mathrm{d} \underline{v} \mid \\
& \leq 2 \int_{\mathbb{R}^{2}} \boldsymbol{g}_{f}(\underline{v}) \sup _{y \in \mathbb{R}^{2}}\left|\int_{\mathbb{R}^{2}} K(\bar{v}) \boldsymbol{g}_{f}\left(y-\left[\boldsymbol{p}_{2}-\boldsymbol{p}_{1}^{2} \boldsymbol{p}_{2}^{-1}\right] \bar{v} h\right) \mathrm{d} \bar{v}-\boldsymbol{g}_{f}(y)\right| \mathrm{d} \underline{v} \\
& \leq 2 C(\mathcal{K}, \beta, 1) L^{2} h^{\beta} .
\end{aligned}
$$

Here we have also used that $\boldsymbol{p}_{1}^{2}+\boldsymbol{p}_{2}^{2}=1$ and therefore $\left(\boldsymbol{p}_{1} \vee \boldsymbol{p}_{2}\right)^{2} \geq 1 / 2$.

imsart-generic ver. 2014/10/16 file: rotation-arXiv.tex date: February 26, 2020 
$\mathbf{2}^{\mathbf{0}} \mathbf{b}$ If $\left|\boldsymbol{p}_{2}\right|<\left|\boldsymbol{p}_{1}\right|$ making the change of variables $\bar{t}=\bar{v}$ and $\boldsymbol{p}_{2} \bar{t} h+\boldsymbol{p}_{1} \Gamma \underline{t}=$ $\underline{v}$, we obtain

$$
\begin{aligned}
& \mathcal{E}_{h}(f, D, x) \\
& =\boldsymbol{p}_{1}^{-2} \int_{\mathbb{R}^{4}} \boldsymbol{g}_{f}(\underline{v}) K(\bar{v}) \boldsymbol{g}_{f}\left(\boldsymbol{p}_{1}^{-1} D \Omega x+\left[\boldsymbol{p}_{1}-\boldsymbol{p}_{2}^{2} \boldsymbol{p}_{1}^{-1}\right] \Omega \bar{v} h+\boldsymbol{p}_{2} \boldsymbol{p}_{1}^{-1} \Omega \underline{v}\right) \mathrm{d} v .
\end{aligned}
$$

We deduce from Lemma 4 similarly to (4.2)

$$
\begin{aligned}
& \left|\mathcal{E}_{h}(f, D, x)-\boldsymbol{p}_{1}^{-2} \int_{\mathbb{R}^{2}} \boldsymbol{g}_{f}(\underline{v}) \boldsymbol{g}_{f}\left(\boldsymbol{p}_{1}^{-1} D \Omega x+\boldsymbol{p}_{2} \boldsymbol{p}_{1}^{-1} \Omega \underline{v}\right) \mathrm{d} v\right| \\
& \leq 2 C(\mathcal{K}, \beta, 1) L^{2} h^{\beta} .
\end{aligned}
$$

It is worth noting that (4.2) and (4.3) can be written in a unified way

$$
\left|\mathcal{E}_{h}(f, D, x)-\mathcal{E}_{0}(f, D, x)\right| \leq 2 C(\mathcal{K}, \beta, 1) L^{2} h^{\beta} .
$$

Thus, remarking that $\tau_{f}\left(D, \boldsymbol{Q}_{f}\right)=\mathcal{E}_{0}(f, D, x)$ we come to the assertion of the lemma.

Proof of Lemma 2. Since by definition $\widetilde{f}_{h,\left(\boldsymbol{Q}_{f}, \boldsymbol{Q}_{f}\right)}(x)=\widetilde{f}_{h, \boldsymbol{Q}_{f}}(x)$ it suffices to prove the lemma for any $D \neq \boldsymbol{Q}_{f}$. We obviously have

$$
\begin{aligned}
& E_{h}(f, D, x):=\mathbb{E}_{f}\left[\widetilde{f}_{h,\left(D, Q_{f}\right)}(x)\right] \\
= & \int_{\mathbb{R}^{4}} K_{h}(z) \boldsymbol{g}_{f}\left(\boldsymbol{Q}_{f}^{T} \bar{y}+\boldsymbol{p}_{1} \boldsymbol{Q}_{f}^{T} \Gamma \Omega \bar{z}+\boldsymbol{p}_{2} \boldsymbol{Q}_{f}^{T} \Omega \underline{z}\right) \boldsymbol{g}_{f}\left(\boldsymbol{p}_{2} \boldsymbol{Q}_{f}^{T} \bar{z}+\boldsymbol{p}_{1} \boldsymbol{Q}_{f}^{T} \Gamma \underline{z}\right) \mathrm{d} z .
\end{aligned}
$$

Noting that

$$
\boldsymbol{Q}_{f}^{T} \Omega \boldsymbol{Q}_{f}^{T}=\Omega, \quad \boldsymbol{Q}_{f}^{T} \Gamma \boldsymbol{Q}_{f}^{T}=\Gamma
$$

and putting $\underline{z}=\boldsymbol{Q}_{f}^{T} \underline{u}, \bar{z}=\bar{u} h$ we get

$$
\begin{aligned}
& E_{h}(f, D, x) \\
& =\int_{\mathbb{R}^{4}} K(\bar{u}) \boldsymbol{g}_{f}\left(\boldsymbol{Q}_{f}^{T} \bar{y}+\boldsymbol{p}_{1} \boldsymbol{Q}_{f}^{T} \Gamma \Omega \bar{u} h+\boldsymbol{p}_{2} \Omega \underline{u}\right) \boldsymbol{g}_{f}\left(\boldsymbol{p}_{2} \boldsymbol{Q}_{f}^{T} \bar{u} h+\boldsymbol{p}_{1} \Gamma \underline{u}\right) \mathrm{d} u .
\end{aligned}
$$

Consider now two cases.

$\mathbf{1}^{\mathbf{0}} \mathbf{a}$. If $\left|\boldsymbol{p}_{2}\right| \geq\left|\boldsymbol{p}_{1}\right|$ using $\Omega^{2}=I, \Gamma \Omega \boldsymbol{Q}_{f}^{T} \Gamma \Omega=-\boldsymbol{Q}_{f}^{T}, \boldsymbol{p}_{1}^{2}+\boldsymbol{p}_{2}^{2}=1$ and making the change of variables $\bar{u}=\bar{v}$,

$\boldsymbol{Q}_{f}^{T} \bar{y}+\boldsymbol{p}_{1} \boldsymbol{Q}_{f}^{T} \Gamma \Omega \bar{u} h+\boldsymbol{p}_{2} \Omega \underline{u}=\underline{v} \Rightarrow \underline{u}=\boldsymbol{p}_{2}^{-1} \Omega \underline{v}-\boldsymbol{p}_{2}^{-1} \Omega \boldsymbol{Q}_{f}^{T} y-\boldsymbol{p}_{1} \boldsymbol{p}_{2}^{-1} \Omega \boldsymbol{Q}_{f}^{T} \Gamma \Omega \bar{v} h$ we obtain 


$$
\begin{aligned}
& E_{h}(f, D, x) \\
& =\boldsymbol{p}_{2}^{-2} \int_{\mathbb{R}^{4}} \boldsymbol{g}_{f}(\underline{v}) K(\bar{v}) \boldsymbol{g}_{f}\left(-\boldsymbol{p}_{1} \boldsymbol{p}_{2}^{-1} \boldsymbol{Q}_{f}^{T} \bar{y} \Gamma \Omega+\boldsymbol{p}_{1} \boldsymbol{p}_{2}^{-1} \Gamma \Omega \underline{v}+\boldsymbol{p}_{2}^{-1} \boldsymbol{Q}_{f}^{T} \bar{v} h\right) \mathrm{d} v .
\end{aligned}
$$

Applying Lemma 4 we obtain similarly to (4.2)

$$
\begin{aligned}
& \left|E_{h}(f, D, x)-\boldsymbol{p}_{2}^{-2} \int_{\mathbb{R}^{2}} \boldsymbol{g}_{f}(\underline{v}) \boldsymbol{g}_{f}\left(-\boldsymbol{p}_{1} \boldsymbol{p}_{2}^{-1} \boldsymbol{Q}_{f}^{T} \bar{y} \Gamma \Omega+\boldsymbol{p}_{1} \boldsymbol{p}_{2}^{-1} \Gamma \Omega \underline{v}\right) \mathrm{d} \underline{v}\right| \\
& \leq 2 C(\mathcal{K}, \beta, \sqrt{2}) L^{2} h^{\beta} .
\end{aligned}
$$

$\mathbf{1}^{0} \mathbf{b}$. If $\left|\boldsymbol{p}_{1}\right|>\left|\boldsymbol{p}_{2}\right|$ using $\Gamma^{2}=I, \boldsymbol{Q}_{f}^{T} \Gamma \Omega=-\Omega \Gamma \boldsymbol{Q}_{f}^{T}, \boldsymbol{p}_{1}^{2}+\boldsymbol{p}_{2}^{2}=1$ and making the change of variables $\bar{u}=\bar{v}$,

$$
\boldsymbol{p}_{2} \boldsymbol{Q}_{f}^{T} \bar{u} h+\boldsymbol{p}_{1} \Gamma \underline{u}=\underline{v} \Rightarrow \underline{u}=\boldsymbol{p}_{1}^{-1} \Gamma \underline{v}-\boldsymbol{p}_{2} \boldsymbol{p}_{1}^{-1} \Gamma \boldsymbol{Q}_{f}^{T} \bar{v} h
$$

we obtain

$$
\begin{aligned}
& E_{h}(f, D, x) \\
& =\boldsymbol{p}_{1}^{-2} \int_{\mathbb{R}^{4}} \boldsymbol{g}_{f}(\underline{v}) K(\bar{v}) \boldsymbol{g}_{f}\left(\boldsymbol{Q}_{f}^{T} \bar{y}+\boldsymbol{p}_{2} \boldsymbol{p}_{1}^{-1} \Omega \Gamma \underline{v}+\boldsymbol{p}_{1}^{-1} \boldsymbol{Q}_{f}^{T} \Gamma \Omega \bar{v} h\right) \mathrm{d} v .
\end{aligned}
$$

The application of Lemma 4 yields

$$
\begin{aligned}
& \left|E_{h}(f, D, x)-\boldsymbol{p}_{1}^{-2} \int_{\mathbb{R}^{2}} \boldsymbol{g}_{f}(\underline{v}) \boldsymbol{g}_{f}\left(\boldsymbol{Q}_{f}^{T} \bar{y}+\boldsymbol{p}_{2} \boldsymbol{p}_{1}^{-1} \Omega \Gamma \underline{v}\right) \mathrm{d} \underline{v}\right| \\
& \leq 2 C(\mathcal{K}, \beta, 1) L^{2} h^{\beta} \leq 2 C(\mathcal{K}, \beta, \sqrt{2}) L^{2} h^{\beta} .
\end{aligned}
$$

Note that (4.4) and (4.5) can be written as

$$
\left|E_{h}(f, D, x)-E_{0}(f, D, x)\right| \leq 2 C(\mathcal{K}, \beta, \sqrt{2}) L^{2} h^{\beta},
$$

Note also that $\boldsymbol{Q}_{f}^{T} \bar{y}=\boldsymbol{p}_{1}^{-1} D \Omega x$ and

$$
\begin{aligned}
E_{0}(f, D, x) & =\int_{\mathbb{R}^{2}} \boldsymbol{g}_{f}\left(\boldsymbol{p}_{1}^{-1} D \Omega x+\boldsymbol{p}_{2} \Omega \underline{u}\right) \boldsymbol{g}_{f}\left(\boldsymbol{p}_{1} \Gamma \underline{u}\right) \mathrm{d} \underline{u} \\
& =\int_{\mathbb{R}^{2}} \boldsymbol{g}_{f}\left(\boldsymbol{p}_{1}^{-1} D \Omega x+\boldsymbol{p}_{2} \Omega \Gamma u\right) \boldsymbol{g}_{f}\left(\boldsymbol{p}_{1} \Gamma u\right) \mathrm{d} u=\tau_{f}\left(D, \boldsymbol{Q}_{f}\right) .
\end{aligned}
$$

To get the penultimate equality we used the change of variables $u_{1}=\underline{v}_{1}$, $u_{2}=-\underline{v}_{2}$ and the symmetry of $\boldsymbol{g}_{f, 2}$ which implies $g\left(p_{1} v\right)=g\left(p_{1} \Gamma v\right)$. Hence,

$$
\left|\mathbb{E}_{f}\left[\widetilde{f}_{h,\left(D, \boldsymbol{Q}_{f}\right)}(x)\right]-\tau_{f}\left(D, \boldsymbol{Q}_{f}\right)\right| \leq 2 C(\mathcal{K}, \beta, \sqrt{2}) L^{2} h^{\beta}
$$

that implies together with Lemma 1 the assertion of the lemma.

imsart-generic ver. 2014/10/16 file: rotation-arXiv.tex date: February 26, 2020 
Proof of Lemma 3. As it was mentioned in Remark $1 p_{1}(D, Q)=-p_{1}(Q, D)$ and $p_{2}(D, Q)=p_{2}(Q, D)$. Moreover $D Q=Q D$ for any $D, Q \in \mathfrak{Q}$. Hence

$$
\begin{aligned}
\tilde{f}_{h,(Q, D)}(x) & =\frac{1}{n(n-1)} \sum_{k, l=1, k \neq l}^{n} K_{h}\left(-p_{1} \Omega \Gamma X_{k}+p_{2} X_{l}-\Gamma \Omega Q D \Omega x\right) \\
& =\frac{1}{n(n-1)} \sum_{k, l=1, k \neq l}^{n} K_{h}\left(p_{1} \Omega \Gamma\left(-X_{k}\right)+p_{2} X_{l}-\Gamma \Omega Q D \Omega x\right)
\end{aligned}
$$

Remind that the density of $X_{k}$ is $\boldsymbol{g}_{f}\left(\boldsymbol{Q}_{f}^{T} v\right)$ and therefore the law of $-X_{k}$ coincides with whose of $X_{k}$ because $\boldsymbol{g}_{f}$ is symmetric. Finally since $X_{k}$ and $X_{l}$ are independent for all $k \neq l$ for any $D, Q \in \mathfrak{Q}$ we conclude that

$$
K_{h}\left(-p_{1} \Omega \Gamma X_{k}+p_{2} X_{l}-\Gamma \Omega Q D \Omega x\right) \stackrel{\text { law }}{=} K_{h}\left(p_{1} \Omega \Gamma X_{k}+p_{2} X_{l}-\Gamma \Omega Q D \Omega x\right) .
$$

It implies in particular the assertion of the lemma.

Proof of Proposition 1. Denoting $\left(\xi_{1, i}, \xi_{2, i}\right)^{T}=\boldsymbol{Q}_{f}^{T}\left(X_{i}-x\right), i=1, \ldots, n$, we remark that

$$
\widetilde{f}_{\mathfrak{h}, \boldsymbol{Q}_{f}}^{(\mathcal{I})}(x)=\left[|\mathcal{I}|^{-1} \sum_{k \in \mathcal{I}} \mathcal{K}_{\boldsymbol{h}}\left(\xi_{1, k}\right)\right]\left[|\mathcal{I}|^{-1} \sum_{k \in \mathcal{I}} \mathcal{K}_{\boldsymbol{h}}\left(\xi_{2, k}\right)\right]=: \Upsilon_{1}(\boldsymbol{h}) \Upsilon_{2}(\boldsymbol{h}) .
$$

Note that $\xi_{1, i}, \xi_{2, i}, i=1, \ldots, n$ are independent with the densities given by $\boldsymbol{g}_{1}\left(\bullet+\boldsymbol{q}^{T} x\right)$ and $\boldsymbol{g}_{2}\left(\bullet+\boldsymbol{q}_{\perp}^{T} x\right)$ respectively. We obviously have

$$
\begin{aligned}
\widetilde{f}_{\boldsymbol{h}, \boldsymbol{Q}_{f}}^{(\mathcal{I})}(x)-f(x)=[ & \left.\boldsymbol{\Upsilon}_{1}(\boldsymbol{h})-\mathbb{E}_{\boldsymbol{g}_{f}}\left\{\Upsilon_{1}(\boldsymbol{h})\right\}\right]\left[\Upsilon_{2}(\mathfrak{h})-\mathbb{E}_{\boldsymbol{g}_{f}}\left\{\Upsilon_{2}(\boldsymbol{h})\right\}\right] \\
& +\mathbb{E}_{\boldsymbol{g}_{f}}\left\{\Upsilon_{1}(\boldsymbol{h})\right\}\left[\Upsilon_{2}(\boldsymbol{h})-\mathbb{E}_{\boldsymbol{g}_{f}}\left\{\Upsilon_{2}(\boldsymbol{h})\right\}\right] \\
& +\mathbb{E}_{\boldsymbol{g}_{f}}\left\{\Upsilon_{2}(\boldsymbol{h})\right\}\left[\Upsilon_{1}(\boldsymbol{h})-\mathbb{E}_{\boldsymbol{g}_{f}}\left\{\Upsilon_{1}(\boldsymbol{h})\right\}\right] \\
& +\mathbb{E}_{\boldsymbol{g}_{f}}\left\{\Upsilon_{1}(\boldsymbol{h})\right\} \mathbb{E}_{\boldsymbol{g}_{f}}\left\{\Upsilon_{2}(\boldsymbol{h})\right\}-f(x) .
\end{aligned}
$$

Here $\mathbb{E}_{\boldsymbol{g}_{f}}$ is the expectation w.r.t the law of $\xi_{1}, \ldots, \xi_{n}$. In view of Lemma 1

$$
\begin{aligned}
& \left|\mathbb{E}_{\boldsymbol{g}_{f}}\left\{\Upsilon_{1}(\boldsymbol{h})\right\} \mathbb{E}_{\boldsymbol{g}_{f}}\left\{\Upsilon_{1}(\boldsymbol{h})\right\}-f(x)\right|=\left|\mathbb{E}_{\boldsymbol{g}_{f}}\left\{\mathcal{K}_{\boldsymbol{h}}\left(\xi_{1,1}\right)\right\} \mathbb{E}_{\boldsymbol{g}_{f}}\left\{\mathcal{K}_{\boldsymbol{h}}\left(\xi_{2,1}\right)\right\}-f(x)\right| \\
& \leq 2 C(\mathcal{K}, \beta, 1) L^{2} \boldsymbol{h}^{\beta}=2 C(\mathcal{K}, \beta, 1) L^{2}(\mu /|\mathcal{I}|)^{\frac{\beta}{2 \beta+1}} .
\end{aligned}
$$

Since $\boldsymbol{g}_{f} \in \mathcal{G}(\beta, L)$ it implies $\boldsymbol{g}_{1}, \boldsymbol{g}_{2}$ are uniformly bounded by $L$. Hence

$$
\begin{aligned}
& \left|\mathbb{E}_{\boldsymbol{g}_{f}}\left\{\Upsilon_{j}(\boldsymbol{h})\right\}\right| \leq L\|\mathcal{K}\|_{1}, \quad \mathbb{V}_{\boldsymbol{g}_{f}}\left\{|\mathcal{I}| \Upsilon_{j}(\boldsymbol{h})\right\} \leq L\|\mathcal{K}\|_{2}^{2}|\mathcal{I}| \boldsymbol{h}^{-1}, j=1,2 ; \\
& \mathbb{E}_{\boldsymbol{g}_{f}}\left\{\mid \mathcal{K}_{\boldsymbol{h}}\left(\left.\xi_{1, k}\right|^{p}\right\} \leq L\|\mathcal{K}\|_{p}^{p} \boldsymbol{h}^{1-p}, \quad \mathbb{E}_{\boldsymbol{g}_{f}}\left\{\mid \mathcal{K}_{\boldsymbol{h}}\left(\left.\xi_{2, k}\right|^{p}\right\} \leq L\|\mathcal{K}\|_{p}^{p} \boldsymbol{h}^{1-p} .\right.\right.
\end{aligned}
$$


Applying the Rosenthal inequality (if $p>2$ ) to $|\mathcal{I}|\left[\Upsilon_{j}(\mathfrak{h})-\mathbb{E}_{\boldsymbol{g}_{f}}\left\{\Upsilon_{j}(\mathfrak{h})\right\}\right], j=$ 1,2 , which is a sum of i.i.d bounded and centered random variables or computing its variance (if $1 \leq p \leq 2$ ) we assert that there exists $C>0$ completely determined by $p$ and $\mathcal{K}$ such that for any $n \geq 1, \mathcal{I} \in \mathfrak{I}_{n}$ and $\mu \geq 1$

$$
\mathbb{E}_{\boldsymbol{g}_{f}}\left\{\left|\Upsilon_{j}(\boldsymbol{h})\right|^{p}\right\} \leq C\left(L^{p / 2}+L\right)(\mu /|\mathcal{I}|)^{\frac{\beta}{2 \beta+1}}, j=1,2 .
$$

The assertion of the proposition follows now from (4.6) and (4.7).

Proof of Lemma 4 Remind that for any function $w \in \mathbb{H}(\beta, L)$

$$
\sup _{z, \mathfrak{z} \in \mathbb{R}}|z-\mathfrak{z}|^{-\beta}\left|\sum_{j=0}^{m} \frac{w^{(j)}(\mathfrak{z})(z-\mathfrak{z})^{j}}{j !}-w(z)\right| \leq L .
$$

We deduce from (4.8) for any $t \in \mathbb{R}^{2}$

$$
\begin{aligned}
& \left|g_{1}\left(y_{1}+h \psi_{1}^{T} t\right)-\sum_{j=0}^{m} \frac{g_{1}^{(j)}\left(y_{1}\right) h^{j}\left(\psi_{1}^{T} t\right)^{j}}{j !}\right| \leq L h^{\beta}\left|\psi_{1}^{T} t\right|^{\beta} \leq \psi^{*} L h^{\beta}\|t\|^{\beta} ; \\
& \left|g_{2}\left(y_{2}+h \psi_{2}^{T} t\right)-\sum_{j=0}^{m} \frac{g_{2}^{(j)}\left(y_{2}\right) h^{j}\left(\psi_{2}^{T} t\right)^{j}}{j !}\right| \leq L h^{\beta}\left|\psi_{2}^{T} t\right|^{\beta} \leq \psi^{*} L h^{\beta}\|t\|^{\beta},
\end{aligned}
$$

where $\|\cdot\|$ is used for the euclidian norm. Setting

$$
\mathbf{P}_{g, \Psi, y}(t)=\sum_{j, s=0}^{m} \frac{g_{1}^{(j)}\left(y_{1}\right) g_{2}^{(s)}\left(y_{2}\right) h^{j+s}\left(\psi_{1}^{T} t\right)^{j}\left(\psi_{2}^{T} t\right)^{s}}{j ! s !}
$$

and recalling that $\left\|g_{i}\right\|_{\infty} \leq L, i=1,2$, we obviously have

$$
\left|g(y+\Psi t h)-\mathbf{P}_{g, \Psi, y}(t)\right| \leq 2 \psi^{*} L^{2} h^{\beta}\|t\|^{\beta}+\left(\psi^{*} L\right)^{2} h^{2 \beta}\|t\|^{2 \beta} .
$$

It remains to note that $\mathbf{P}_{g, \Psi, y}(t)$ can be rewritten as

$$
\mathbf{P}_{g, \Psi, y}(t)=\sum_{i, l=0}^{2 m} a_{i, l} t_{1}^{i} t_{2}^{l}, \quad a_{0,0}=g_{1}\left(y_{1}\right) g_{2}\left(y_{2}\right)=g(y),
$$

and, therefore, in view of Assumption 1

$$
\int_{\mathbb{R}^{2}} K(t) \mathbf{P}_{g, \Psi, y}(t) \mathrm{d} t=g(y) .
$$

This together with (4.9) allows as to assert that

$$
\left|\int_{\mathbb{R}^{2}} K(t) g(y+\Psi t h) \mathrm{d} t-g(y)\right| \leq C\left(\mathcal{K}, \beta, \psi^{*}\right) L^{2} h^{\beta}, \quad \forall h>0 .
$$

Lemma is proved.

imsart-generic ver. 2014/10/16 file: rotation-arXiv.tex date: February 26, 2020 


\section{Proofs of Propositions 2-3}

Set $\beta_{1}, \beta_{2}>0, L \geq 1$ and let $f \in \mathcal{F}\left(\beta, L, \mathcal{Q}_{\delta_{n}}\right), \beta \in\left\{\beta_{1}, \beta_{2}\right\}$, be fixed. We divide these proofs into three steps.

First step: upper bounds for sums of independent variables.

For any $(h, D) \in \mathbb{R}_{+}^{*} \times \mathcal{Q}_{\delta_{n}}$ and any $b \in\left\{d, d_{\perp}\right\}$ set

$$
\begin{aligned}
& \xi_{h, D}(x):=\widetilde{f}_{h, D}(x)-\mathbb{E}_{f}\left\{\widetilde{f}_{(h, d)}(x)\right\} \mathbb{E}_{f}\left\{\widetilde{f}_{\left(h, d_{\perp}\right)}(x)\right\}, \\
& \xi_{(h, b)}(x):=\widetilde{f}_{(h, b)}(x)-\mathbb{E}_{f}\left\{\widetilde{f}_{(h, b)}(x)\right\}, \\
& G_{h, b}(x):=1 \vee \mathbb{E}_{f}\left\{\left|\mathcal{K}_{h}\left(b^{T} X_{1}-b^{T} x\right)\right|\right\} \\
& \widetilde{G}_{h, b}(x):=1 \vee\left[\frac{1}{n} \sum_{k=1}^{n}\left|\mathcal{K}_{h}\left(b^{T} X_{k}-b^{T} x\right)\right|\right] .
\end{aligned}
$$

Note first that, since $\left|\boldsymbol{q}^{T} b\right|^{2}+\left|\boldsymbol{q}^{T} b_{\perp}\right|^{2}=1$ and $L \wedge\|\mathcal{K}\|_{1} \geq 1$,

$$
\begin{aligned}
& G_{h, b}(x)=1 \vee \int_{\mathbb{R}^{2}}\left|\mathcal{K}\left(v_{1}\right)\right| \boldsymbol{g}_{1}\left(\boldsymbol{q}^{T} x+h \boldsymbol{q}^{T} b v_{1}+\boldsymbol{q}^{T} b_{\perp} v_{2}\right) \times \\
& \boldsymbol{g}_{2}\left(\boldsymbol{q}_{\perp}^{T} x+h \boldsymbol{q}_{\perp}^{T} b v_{1}+\boldsymbol{q}_{\perp}^{T} b_{\perp} v_{2}\right) \mathrm{d} v \leq \sqrt{2}\|\mathcal{K}\|_{1} L .
\end{aligned}
$$

For any $q \geq 1$ and any $\epsilon>0$ put $\lambda_{q}^{(1)}(\epsilon)=\left[\sqrt{2}+\sqrt{5 q \epsilon^{-1}}\right]\left(\|\mathcal{K}\|_{\infty} \vee 1\right)$. Consider finally a real number $\alpha_{n} \geq 1 \vee \mathfrak{n}\left(\mathcal{Q}_{\delta_{n}}\right)$. In the sequel $\alpha_{n}$ and $\epsilon$ will be fixed and properly chosen.

Applying Bernstein inequality we obtain for any $q \geq 2$, any integer $n \geq 3$, any $z \in\left[0,2 q \alpha_{n}\right]$ and all real numbers $h$ satisfying $n h \geq \epsilon \alpha_{n}$

$$
\begin{array}{r}
\sup _{b \in\left\{d, d_{\perp}\right\}} \mathbb{P}_{f}\left\{\sup _{D \in \mathcal{Q}_{\delta_{n}}}\left[\left|\xi_{(h, b)}(x)\right|-\lambda_{q}^{(1)}(\epsilon) G_{h, b}(x) \sqrt{\frac{0.5 q \alpha_{n}+z}{n h}}\right]>0\right\} \\
\leq 2 e^{-z} .
\end{array}
$$

By integration of the Bernstein inequality we get for any $q \geq 1, n \geq 3$, any $t \in\left[0,1.5 q \alpha_{n}\right]$ and any real $h$ satisfying $n h \geq \epsilon \alpha_{n}$

$$
\begin{array}{r}
\sup _{b \in\left\{d, d_{\perp}\right\}} \mathbb{E}_{f}\left\{\sup _{D \in \mathcal{Q}_{\delta_{n}}}\left[\left|\xi_{(h, b)}(x)\right|-\lambda_{q}^{(1)}(\epsilon) G_{h, b}(x) \sqrt{\frac{q \alpha_{n}+t}{n h}}\right]_{+}\right\}^{q} \\
\leq C_{1}^{(q)}(\mathcal{K}) L^{q}[n h]^{-\frac{q}{2}} e^{-t}
\end{array}
$$

where $C_{1}^{(q)}(\mathcal{K}, \epsilon)=2^{q+1}\left(1+\sqrt{\epsilon^{-1}}\right)^{q} \Gamma(q+1)\|\mathcal{K}\|_{1}^{q}\left(\|\mathcal{K}\|_{\infty} \vee 1\right)^{q}$ and $\Gamma$ is the Gamma function. Choose now $\alpha_{n}=\alpha \ln (n), \epsilon=1$ and $t=1,5 q \alpha_{n}$ and introduce $\gamma_{q}=\lambda_{q}^{(1)}(1) \sqrt{2.5 q \alpha}$, 


$$
\mathfrak{H}_{q}:=\left\{h \in \mathcal{H}: n h \geq 10 q\left[\lambda_{q}^{(1)}(1)\right]^{2} \alpha_{n}\right\} .
$$

Since $\operatorname{card}\left(\mathfrak{H}_{q}\right) \leq \ln (n)$, we deduce from (5.2) that for any $q \geq 1$ and $n \geq 3$

$$
\begin{aligned}
& \sup _{b \in\left\{d, d_{\perp}\right\}} \mathbb{E}_{f}\left\{\sup _{h \in \mathfrak{H}_{q}} \sup _{D \in \mathcal{Q}_{\delta_{n}}}\left[\left|\xi_{(h, b)}(x)\right|-\gamma_{q} G_{h, b}(x) \sqrt{\ln (n) / n h}\right]_{+}\right\}^{q} \\
& \leq C_{1}^{(q)}(\mathcal{K}, 1) L^{q}[\ln (n) / n]^{\frac{q}{2}} n^{-q} .
\end{aligned}
$$

Additionally, using kernel $|\mathcal{K}|$ instead of $\mathcal{K}$ in the last inequality we get

$$
\begin{aligned}
& \sup _{b \in\left\{d, d_{\perp}\right\}} \mathbb{E}_{f}\left\{\sup _{h \in \mathfrak{H}_{q}} \sup _{D \in \mathcal{Q}_{\delta_{n}}}\left[G_{h, b}(x)-2 \widetilde{G}_{h, b}(x)\right]_{+}\right\}^{q} \\
& \leq 2^{q} C_{1}^{(q)}(\mathcal{K}, 1) L^{q}\left[\frac{\ln (n)}{n}\right]^{\frac{q}{2}} n^{-q} \\
& \mathbb{E}_{f}\left\{\sup _{h \in \mathfrak{H}_{q}} \sup _{D \in \mathcal{Q}_{\delta_{n}}} \sup _{b \in\left\{d, d_{\perp}\right\}}\left[\widetilde{G}_{h, b}(x)\right]\right\}^{q} \\
& \leq 2^{q-1}\left[2 C_{1}^{(q)}(\mathcal{K}, 1)+\left(\frac{3}{\sqrt{2}}\right)^{q}\|\mathcal{K}\|_{1}^{q}\right] L^{q} .
\end{aligned}
$$

Noting that $\widehat{\mathcal{U}}_{n}=\sup _{h \in \mathfrak{H}} \sup _{D \in \mathcal{Q}_{\delta_{n}}} \sup _{b \in\left\{d, d_{\perp}\right\}}\left[\widetilde{G}_{h, b}(x)\right]^{2}$ and that $\mathfrak{H} \subset \mathfrak{H}_{2 p}$ for $n$ large enough, we obtain the third assertion of Proposition 3 with

$$
\mathbf{C}_{p}(\mathcal{K})=2^{2 p-1}\left[2 C_{1}^{(2 p)}(\mathcal{K}, 1)+(3 / \sqrt{2})^{2 p}\|\mathcal{K}\|_{1}^{2 p}\right] .
$$

Since $\left|\xi_{h, D}(x)\right| \leq\left|\xi_{(h, d)}(x)\right| \times \widetilde{G}_{h, d_{\perp}}(x)+\left|\xi_{\left(h, d_{\perp}\right)}(x)\right| \times G_{h, d}(x)$, using CauchySchwartz inequality we get for all $p \geq 1$ and all $n$ large enough

$$
\begin{aligned}
& \mathbb{E}_{f}\left\{\sup _{h \in \mathfrak{H}} \sup _{D \in \mathcal{Q}_{\delta_{n}}}\left[\left|\xi_{h, D}(x)\right|-\mathfrak{a} \widehat{\mathcal{U}}_{n} \sqrt{\ln (n) / n h}\right]_{+}\right\}^{p} \\
& \leq C L^{2 p}[\ln (n) / n]^{\frac{p}{2}} n^{-p}, \quad C:=C(p, \mathcal{K})>0 .
\end{aligned}
$$

Similarly, in view of (5.1) with $q=2 p$ and $z=4 p \alpha_{n}$, one has for all $p \geq 1$ and all $n$ large enough

$$
\begin{array}{r}
\sup _{b \in\left\{d, d_{\perp}\right\}} \mathbb{P}_{f}\left\{\sup _{h \in \mathfrak{H}} \sup _{D \in \mathcal{Q}_{\delta_{n}}}\left[\left|\xi_{(h, b)}(x)\right|-\gamma_{2 p} G_{h, b}(x) \sqrt{\ln (n) / n h}\right]>0\right\} \\
\leq 2 \ln (n) n^{-4 p} \\
\mathbb{P}_{f}\left\{\sup _{h \in \mathfrak{H}} \sup _{D \in \mathcal{Q}_{\delta_{n}}}\left[\left|\xi_{h, D}(x)\right|-\mathfrak{a} \widehat{\mathcal{U}}_{n} \sqrt{\ln (n) / n h}\right]>0\right\} \leq 10 \ln (n) n^{-4 p} .
\end{array}
$$

imsart-generic ver. 2014/10/16 file: rotation-arXiv.tex date: February 26, 2020 
Second step: upper bounds for U-Statistics of Order Two.

For any $(D, Q) \in \mathcal{Q}_{\delta_{n}}^{2}, Q \neq D$, and any $h>0$ put

$$
\begin{aligned}
\xi_{h,(D, Q)}(x) & :=\bar{f}_{h,(D, Q)}(x)-\mathbb{E}_{f}\left[\bar{f}_{h,(D, Q)}(x)\right] \text { and } \\
\varphi\left(X_{k}, X_{l}\right) & :=\frac{1}{n(n-1)} K_{h}\left(p_{1} \Omega \Gamma X_{k}+p_{2} X_{l}-\Omega \Gamma Q D \Omega x\right)
\end{aligned}
$$

Let's write $\xi_{h,(D, Q)}(x)=\xi_{h,(D, Q)}^{(1)}(x)+\xi_{h,(D, Q)}^{(2)}(x)+\xi_{h,(D, Q)}^{(3)}(x)$, where

$$
\begin{aligned}
& \xi_{h,(D, Q)}^{(1)}(x):= \sum_{k, l=1, k \neq l}^{n}\left(\varphi\left(X_{k}, X_{l}\right)-\mathbb{E}_{f}\left[\varphi\left(X_{k}, X_{l}\right) \mid X_{l}\right]\right. \\
&\left.-\mathbb{E}_{f}\left[\varphi\left(X_{k}, X_{l}\right) \mid X_{k}\right]+\mathbb{E}_{f}\left[\varphi\left(X_{k}, X_{l}\right)\right]\right), \\
& \xi_{h,(D, Q)}^{(2)}(x):=\sum_{k, l=1, k \neq l}^{n}\left(\mathbb{E}_{f}\left[\varphi\left(X_{k}, X_{l}\right) \mid X_{l}\right]-\mathbb{E}_{f}\left[\varphi\left(X_{k}, X_{l}\right)\right]\right), \\
& \xi_{h,(D, Q)}^{(3)}(x):=\sum_{k, l=1, k \neq l}^{n}\left(\mathbb{E}_{f}\left[\varphi\left(X_{k}, X_{l}\right) \mid X_{k}\right]-\mathbb{E}_{f}\left[\varphi\left(X_{k}, X_{l}\right)\right]\right) .
\end{aligned}
$$

Note that $\xi_{h,(D, Q)}^{(j)}(x)=\sum_{l=1}^{n}\left(L_{f}^{(j)}\left(X_{l}\right)-\mathbb{E}_{f}\left[L_{f}^{(j)}\left(X_{l}\right)\right]\right), j=2,3$, where

$$
\begin{aligned}
& L_{f}^{(2)}\left(X_{l}\right):=\int_{\mathbb{R}^{2}} n^{-1} K_{h}\left(p_{1} \Omega \Gamma y+p_{2} X_{l}-\Omega \Gamma Q D \Omega x\right) f(y) \mathrm{d} y, \\
& L_{f}^{(3)}\left(X_{l}\right):=\int_{\mathbb{R}^{2}} n^{-1} K_{h}\left(p_{1} \Omega \Gamma X_{l}+p_{2} y-\Omega \Gamma Q D \Omega x\right) f(y) \mathrm{d} y, \\
& \left|L_{f}^{(j)}\left(X_{l}\right)\right| \leq L^{2}\|\mathcal{K}\|_{1}^{2}\left(n \delta_{n}^{2}\right)^{-1} \text { and } \\
& \sum_{l=1}^{n} \operatorname{Var}_{f}\left[L_{f}^{(j)}\left(X_{l}\right)\right] \leq 4 L^{4}\left(\|\mathcal{K}\|_{1}^{4} \vee\|\mathcal{K}\|_{2}^{4}\right) n^{-1}, \text { since } p_{1}^{2}+p_{2}^{2}=1 .
\end{aligned}
$$

Put $\lambda_{p}^{(2)}(\epsilon):=2 \sqrt{\epsilon^{-1}}[\sqrt{2}+\sqrt{3 p}]\left(\|\mathcal{K}\|_{1}^{2} \vee\|\mathcal{K}\|_{2}^{2}\right)$.

As for $j=2,3$ the $L_{f}^{(j)}\left(X_{l}\right)$ 's are independent variables, we get from Bernstein inequality that for any $p \geq 1$, integer $n \geq 3$, any $z>0$ satisfying 
$0 \leq z \leq 4 p \alpha_{n}$ and any $h>0$ satisfying $h \leq \epsilon^{-1}$ and $\epsilon \alpha_{n} h \leq n \delta_{n}^{4}$

$$
\begin{array}{r}
\sup _{j=2,3} \mathbb{P}_{f}\left\{\sup _{D, Q \in \mathcal{Q}_{\delta_{n}}, Q \neq D}\left[\left|\xi_{h,(D, Q)}^{(j)}(x)\right|-\lambda_{p}^{(2)}(\epsilon) L^{2} \sqrt{\frac{2 p \alpha_{n}+z}{n h}}\right]>0\right\} \\
\sup _{j=2,3} \mathbb{E}_{f}\left\{\sup _{D, Q \in \mathcal{Q}_{\delta_{n}}, Q \neq D}\left[\left|\xi_{h,(D, Q)}^{(j)}(x)\right|-\lambda_{p}^{(2)}(\epsilon) L^{2} \sqrt{\frac{2 p \alpha_{n}+z}{n h}}\right]_{+}\right\}^{p} \\
\leq C_{2}^{(p)}(\mathcal{K}, \epsilon) L^{2 p}[n h]^{-\frac{p}{2}} e^{-z}
\end{array}
$$

where $C_{2}^{(p)}(\mathcal{K}, \epsilon)=2^{\frac{p}{2}+1}\left(3 \sqrt{\epsilon^{-1}}\right)^{p} \Gamma(p+1)\left(\|\mathcal{K}\|_{1}^{2} \vee\|\mathcal{K}\|_{2}^{2}\right)^{p}$

On the other hand, choosing $\epsilon=1$ and $\alpha_{n}=\alpha \ln (n)$, one has, in view of (2.3), for all $p \geq 1$ and all integer $n \geq 3$

$\mathbb{P}_{f}\left\{\sup _{h \in \mathfrak{H} D, Q \in \mathcal{Q}_{\delta_{n}}, Q \neq D}\left[\left|\xi_{h,(D, Q)}^{(j)}(x)\right|-\alpha \sqrt{6 p} \lambda_{p}^{(2)}(1) L^{2} \sqrt{\ln (n) / n}\right]>0\right\}$ $\leq 2 \ln (n) n^{-4 p}, j=2,3$, and then

$$
\begin{aligned}
& \sup _{j=2,3} \mathbb{P}_{f}\left\{\sup _{h \in \mathfrak{H} D, Q \in \mathcal{Q}_{\delta_{n}}, Q \neq D}\left[\left|\xi_{h,(D, Q)}^{(j)}(x)\right|-\frac{1}{3} \widehat{\mathcal{U}}_{n} \sqrt{\ln (n) / n h}\right]>0\right\} \\
& \leq 2 \ln (n) n^{-4 p}+\mathbb{P}_{f}\left\{\alpha \sqrt{6 p} \lambda_{p}^{(2)}(1) L^{2} \sqrt{1 / \ln (\ln (n))}>\frac{1}{3} \widehat{\mathcal{U}}_{n} \geq \frac{1}{3}\right\}
\end{aligned}
$$

where the second term of the right hand side is equal to zero for $n$ large enough. Similarly we get for all $p \geq 1$ and all integer $n$ large enough

$$
\begin{array}{r}
\sup _{j=2,3} \mathbb{E}_{f}\left\{\sup _{h \in \mathfrak{H} D, Q \in \mathcal{Q}_{\delta_{n}}, Q \neq D}\left[\left|\xi_{h,(D, Q)}^{(j)}(x)\right|-\frac{1}{3} \widehat{\mathcal{U}}_{n} \sqrt{\ln (n) / n h}\right]_{+}\right\}^{p} \\
\leq C_{2}^{(p)}(\mathcal{K}, 1) L^{2 p}[\ln (n)]^{p} n^{-4 p} .
\end{array}
$$

Now we derive upper bound of $\xi_{h,(D, Q)}^{(1)}(x)$ from exponential inequalities developed in Houdré and Reynaud-Bouret (2003), Theorem 3.4.

$$
\begin{aligned}
& \text { Set } \xi_{h,(D, Q)}^{(1)}(x)=\sum_{k=2}^{n} \sum_{l=1}^{k-1} g\left(X_{k}, X_{l}\right), \text { where } \\
& g\left(X_{k}, X_{l}\right):=\varphi\left(X_{k}, X_{l}\right)+\varphi\left(X_{l}, X_{k}\right)-\mathbb{E}_{f}\left[\varphi\left(X_{k}, X_{l}\right)+\varphi\left(X_{l}, X_{k}\right) \mid X_{l}\right] \\
& \quad-\mathbb{E}_{f}\left[\varphi\left(X_{k}, X_{l}\right)+\varphi\left(X_{l}, X_{k}\right) \mid X_{k}\right]+\mathbb{E}_{f}\left[\varphi\left(X_{k}, X_{l}\right)+\varphi\left(X_{l}, X_{k}\right)\right] .
\end{aligned}
$$

imsart-generic ver. 2014/10/16 file: rotation-arXiv.tex date: February 26, 2020 
Note that $\mathbb{E}_{f}\left[g\left(X_{k}, X_{l}\right) \mid X_{l}\right]=\mathbb{E}_{f}\left[g\left(X_{k}, X_{l}\right) \mid X_{k}\right]=0$ and

$$
\begin{aligned}
\left|g\left(X_{k}, X_{l}\right)\right| & \leq 12\left(1 \vee\|\mathcal{K}\|_{\infty}\right)^{2}(n h)^{-2}=: A, \\
\sum_{k=2}^{n} \sum_{l=1}^{k-1} \mathbb{E}_{f}\left[g\left(X_{k}, X_{l}\right)^{2}\right] & \leq 90\left(\|\mathcal{K}\|_{1}^{4} \vee\|\mathcal{K}\|_{2}^{4}\right) L^{4}(n h)^{-2}=: C^{2} .
\end{aligned}
$$

Moreover for any $a_{k}(\cdot), b_{k}(\cdot), k \in \mathbb{N}^{*}$, verifying $\mathbb{E}_{f}\left[\sum_{k=2}^{n} a_{k}\left(X_{k}\right)^{2}\right] \leq 1$ and $\mathbb{E}_{f}\left[\sum_{l=1}^{n-1} b_{l}\left(X_{l}\right)^{2}\right] \leq 1$ one has using $2 a b \leq a^{2}+b^{2}$

$$
\begin{aligned}
\mathbb{E}_{f}\left[\sum_{k=2}^{n} \sum_{l=1}^{k-1} g\left(X_{k}, X_{l}\right) a_{k}\left(X_{k}\right) b_{l}\left(X_{l}\right)\right] & \leq 4(n-1) \sup _{u \in \mathbb{R}^{2}} \mathbb{E}_{f}\left|\varphi\left(u, X_{1}\right)+\varphi\left(X_{1}, u\right)\right| \\
& \leq 8 L^{2}\|\mathcal{K}\|_{1}^{2}\left(n \delta_{n}^{2}\right)^{-1}=: D .
\end{aligned}
$$

By independence of the $X_{k}$ 's one has for any $u \in \mathbb{R}^{2}$

$$
\begin{aligned}
& \sum_{l=1}^{k-1} \mathbb{E}_{f}\left[g\left(u, X_{l}\right)^{2} \mid X_{k}\right] \\
& =\sum_{l=1}^{k-1} \operatorname{Var}_{f}\left[\left(\varphi\left(u, X_{l}\right)+\varphi\left(X_{l}, u\right)\right)-(n-1)^{-1}\left(L_{f}^{(2)}\left(X_{l}\right)+L_{f}^{(3)}\left(X_{l}\right)\right)\right] \\
& \leq 24 L^{4}\left(\|\mathcal{K}\|_{1}^{4} \vee\|\mathcal{K}\|_{2}^{4}\right) / n^{3} h^{2} \delta_{n}^{2} .
\end{aligned}
$$

Similarly, one has

$$
\sup _{l=1, \ldots, n-1} \sup _{u \in \mathbb{R}^{2}}\left\{\sum_{k=l+1}^{n} \mathbb{E}_{f}\left[g\left(X_{k}, u\right)^{2} \mid X_{l}\right]\right\} \leq \frac{24 L^{4}\left(\|\mathcal{K}\|_{1}^{4} \vee\|\mathcal{K}\|_{2}^{4}\right)}{n^{3} h^{2} \delta_{n}^{2}}=: B^{2} .
$$

It gives for any integer $n \geq 3$ and any real number $z>0$

$$
\mathbb{P}_{f}\left\{\left|\xi_{h,(D, Q)}^{(1)}(x)\right| \geq \mathcal{U}(z)\right\} \leq 6 e^{-z},
$$

where $\mathcal{U}(z):=4 \sqrt{2} C \sqrt{z}+8 \sqrt{2} D z+426 B z^{3 / 2}+414 A z^{2}$.

By integration of the latter inequality we obtain for all $p \geq 1$, all integers $n \geq 3$ and any $z \geq 1$

$$
\mathbb{E}_{f}\left\{\left[\left|\xi_{h,(D, Q)}^{(1)}(x)\right|-\mathcal{U}(z)\right]_{+}\right\}^{p} \leq 3 \times 2^{2 p+1} \Gamma(2 p+1)[z \mathcal{U}(1)]^{p} e^{-z} .
$$

Put $\lambda_{p}^{(3)}(\epsilon):=87955 p \sqrt{p}\left(\|\mathcal{K}\|_{1}^{2} \vee\|\mathcal{K}\|_{2}^{2} \vee\|\mathcal{K}\|_{\infty}^{2}\right)\left[\sqrt{\epsilon^{-1}} \vee \epsilon^{-1} \vee \epsilon^{-\frac{3}{2}}\right]$.

It follows that for all $p \geq 1$, all integer $n \geq 3$, all real number $z$ satisfying $0<z \leq 4 p \alpha_{n}$ and all real number $h>0$ satisfying $n h \geq \epsilon \alpha_{n}, \epsilon \alpha_{n} h \leq n \delta_{n}^{4}$ 
and $\epsilon \alpha_{n} \leq n \delta_{n} \sqrt{h}$

$$
\begin{gathered}
\mathbb{P}_{f}\left\{\sup _{D, Q \in \mathcal{Q}_{\delta_{n}}, Q \neq D}\left[\left|\xi_{h,(D, Q)}^{(1)}(x)\right|-\lambda_{p}^{(3)}(\epsilon) L^{2} \sqrt{\frac{2 p \alpha_{n}+z}{n h}}\right]>0\right\} \\
\leq 6 e^{-z} \\
\mathbb{E}_{f}\left\{\sup _{D, Q \in \mathcal{Q}_{\delta_{n}}, Q \neq D}\left[\left|\xi_{h,(D, Q)}^{(1)}(x)\right|-\lambda_{p}^{(3)}(\epsilon) L^{2} \sqrt{\frac{2 p \alpha_{n}+z}{n h}}\right]_{+}\right\}^{p} \\
\leq C_{3}^{(p)}(\mathcal{K}, \epsilon) 6 \times 2^{2 p} \Gamma(2 p+1) L^{2 p}\left[n h / \alpha_{n}\right]^{-\frac{p}{2}} e^{-z} \\
C_{3}^{(p)}(\mathcal{K}, \epsilon)=\left(\|\mathcal{K}\|_{1}^{2} \vee\|\mathcal{K}\|_{2}^{2} \vee\|\mathcal{K}\|_{\infty}^{2}\right)^{p}\left[45038 p\left(\sqrt{\epsilon^{-1}} \vee \epsilon^{-1} \vee \epsilon^{-\frac{3}{2}}\right)\right]^{p}
\end{gathered}
$$

In another hand, as previously, we get for all $p \geq 1$ and all $n$ large enough

$$
\begin{array}{r}
\mathbb{P}_{f}\left\{\sup _{h \in \mathfrak{H} D, Q \in \mathcal{Q}_{\delta_{n}}, Q \neq D} \sup _{Q}\left[\left|\xi_{h,(D, Q)}^{(1)}(x)\right|-\frac{1}{3} \widehat{\mathcal{U}}_{n} \sqrt{\ln (n) / n h}\right]>0\right\} \\
\leq 6 \ln (n) n^{-4 p}, \\
\mathbb{E}_{f}\left\{\sup _{h \in \mathfrak{H} D, Q \in \mathcal{Q}_{\delta_{n}}, Q \neq D}\left[\left|\xi_{h,(D, Q)}^{(1)}(x)\right|-\frac{1}{3} \widehat{\mathcal{U}}_{n} \sqrt{\ln (n) / n h}\right]\right\}^{p} \\
\leq C_{3}^{(p)}(\mathcal{K}, 1) L^{2 p}[\ln (n)]^{p} n^{-4 p} .
\end{array}
$$

Third step: end of proofs of Propositions 2-3.

Remind that third assertion of Proposition 2 is already proved in step one. First and second ones follow from inequalities (5.4), (5.7), (5.12) and (5.3), (5.8), (5.13) respectively, since $\mathfrak{a} \geq 1$.

End of the proof of Proposition 3. Note first that $\mathbf{B} \geq \mathbf{B}_{1} \vee \mathbf{B}_{2} \vee \mathbf{B}_{3}$, where

$$
\begin{aligned}
& \mathbf{B}_{1}=20 p\left[1+\epsilon_{1}^{-1 / 2}\right]\left(\lambda_{2 p}^{(1)}\left(\epsilon_{1}\right)\|\mathcal{K}\|_{1}\right)^{2} L^{2}+8 C(\mathcal{K}, \mathfrak{b}, \sqrt{2}) L^{2} \\
& \mathbf{B}_{2}=6 \sqrt{6 p} \lambda_{p}^{(2)}\left(\epsilon_{2}\right) L^{2}+8 C(\mathcal{K}, \mathfrak{b}, \sqrt{2}) L^{2} \\
& \mathbf{B}_{3}=6 \sqrt{6 p} \lambda_{p}^{(3)}\left(\epsilon_{3}\right) L^{2}+8 C(\mathcal{K}, \mathfrak{b}, \sqrt{2}) L^{2}
\end{aligned}
$$

Note first that for any $\mathbf{i}=1, \ldots, \mathbf{i}^{*}$

$$
L^{2} h_{\mathbf{i}}^{\beta}=\sqrt{\frac{\omega_{\mathbf{i}}}{\mathbf{n}_{\mathbf{i}} h_{\mathbf{i}}}}, \quad \mathbf{n}_{\mathbf{i}} h_{\mathbf{i}} \geq\left[(8+4 \alpha) L^{\frac{2}{\beta}}\right]^{-\frac{2 \beta}{2 \beta+1}} \omega_{\mathbf{i}}, \quad \ln \left(\frac{\omega_{\mathbf{i}-1} \mathbf{n}_{\mathbf{i}}}{\mathbf{n}_{\mathbf{i}-1} \omega_{\mathbf{i}}}\right) \leq 3 \omega_{\mathbf{i}} .
$$


Thus, in view of (5.1) and (5.2) with $\epsilon=\epsilon_{1}=\left[(8+4 \alpha) L^{\frac{2}{\beta}}\right]^{-\frac{2 \beta}{2 \beta+1}}$ and $\alpha_{n}=$ $\omega_{\mathbf{i}}$ one has for all $p \geq 1$ and all integer $n \geq 3$

$$
\begin{aligned}
& \sup _{b \in\left\{d, d_{\perp}\right\}} \mathbb{P}_{f}\left\{\sup _{D \in \mathcal{Q}_{\delta_{n}}}\left[\left|\xi_{\left(h_{\mathbf{i}}, b\right)}^{(\mathbf{i})}(x)\right|-\lambda_{2 p}^{(1)}\left(\epsilon_{1}\right) \sqrt{10 p}\|\mathcal{K}\|_{1} L^{3} h_{\mathbf{i}}^{\beta}\right]>0\right\} \\
& \leq \frac{2}{e^{8}}\left(\frac{\omega_{\mathbf{i}-1} \mathbf{n}_{\mathbf{i}}}{\mathbf{n}_{\mathbf{i}-1} \omega_{\mathbf{i}}}\right)^{\frac{p \beta}{2 \beta+1}}, \\
& \sup _{b \in\left\{d, d_{\perp}\right\}} \mathbb{E}_{f}\left\{\sup _{D \in \mathcal{Q}_{\delta_{n}}}\left[\left|\xi_{\left(h_{\mathbf{i}}, b\right)}^{(\mathbf{i})}(x)\right|-\lambda_{p}^{(1)}\left(\epsilon_{1}\right) \sqrt{2 p}\|\mathcal{K}\|_{1} L^{3} h_{\mathbf{i}}^{\beta}\right]\right\}^{p} \\
& \leq C_{1}^{(p)}\left(\mathcal{K}, \epsilon_{1}\right) L^{3 p} h_{\mathbf{i}}^{p \beta} \text { and } \\
& \sup _{b \in\left\{d, d_{\perp}\right\}} \mathbb{E}_{f}\left\{\sup _{D \in \mathcal{Q}_{\delta_{n}}}\left|\xi_{\left(h_{\mathbf{i}}, b\right)}^{(\mathbf{i})}(x)\right|\right\}^{p} \\
& \leq 2^{p-1}\left[C_{1}^{(p)}\left(\mathcal{K}, \epsilon_{1}\right)+\left(\lambda_{p}^{(1)}\left(\epsilon_{1}\right) \sqrt{2 p}\|\mathcal{K}\|_{1}\right)^{p}\right] L^{3 p} h_{\mathbf{i}}^{p \beta} .
\end{aligned}
$$

By Cauchy-Schwartz inequality, noting that

$\left|\xi_{h_{\mathbf{i}}, D}^{(\mathbf{i})}(x)\right| \leq \sqrt{2}\|\mathcal{K}\|_{1} L\left(\left|\xi_{\left(h_{\mathbf{i}}, d\right)}^{(\mathbf{i})}(x)\right|+\left|\xi_{\left(h_{\mathbf{i}}, d_{\perp}\right)}^{(\mathbf{i})}(x)\right|\right)+\left|\xi_{\left(h_{\mathbf{i}}, d\right)}^{(\mathbf{i})}(x)\right| \times\left|\xi_{\left(h_{\mathbf{i}}, d_{\perp}\right)}^{(\mathbf{i})}(x)\right|$

we easily get for all $p \geq 1$, all $\mathbf{i}=1, \ldots \mathbf{i}^{*}$ and all integer $n \geq 3$

$$
\begin{array}{r}
\mathbb{P}_{f}\left\{\sup _{D \in \mathcal{Q}_{\delta_{n}}}\left[\left|\xi_{h_{\mathbf{i}}, D}^{(\mathbf{i})}(x)\right|-\mathbf{C} L^{2} h_{\mathbf{i}}^{\beta}\right]>0\right\} \leq \frac{8}{e^{8}}\left(\frac{\omega_{\mathbf{i}-1} \mathbf{n}_{\mathbf{i}}}{\mathbf{n}_{\mathbf{i}-1} \omega_{\mathbf{i}}}\right)^{\frac{p \beta}{2 \beta+1}} \text { and } \\
\mathbb{E}_{f}\left\{\sup _{D \in \mathcal{Q}_{\delta_{n}}}\left[\left|\xi_{h_{\mathbf{i}}, D}^{(\mathbf{i})}(x)\right|-\mathbf{C} L^{2} h_{\mathbf{i}}^{\beta}\right]_{+}\right\}^{p} \leq C^{\prime} h_{\mathbf{i}}^{p \beta},
\end{array}
$$

where $C^{\prime}:=C^{\prime}(p, \mathcal{K}, \beta, L, \alpha)>0$ and $\mathbf{C}=2^{-1} \mathbf{B}-4 C(\mathcal{K}, \mathfrak{b}, \sqrt{2}) L^{2}$.

Remark now that for any $\mathbf{i}=1, \ldots, \mathbf{i}^{*}$ one has $h_{\mathbf{i}} \leq\left[L^{-4}(8+4 \alpha)\right]^{\frac{1}{2 \beta+1}}$ and

$$
\omega_{\mathbf{i}} h_{\mathbf{i}} \leq L^{-\frac{4}{2 \beta+1}}(9+3 \alpha)^{\frac{2 \beta+2}{2 \beta+1}} C(\beta) \mathbf{n}_{\mathbf{i}} \delta_{n}^{4} .
$$

Thus, in view of $(5.5)$ and $(5.6)$ with $\epsilon=\epsilon_{2}=L^{\frac{4}{2 \beta+1}}(9+4 \alpha)^{-\frac{2 \beta+2}{2 \beta+1}}[C(\beta)]^{-1}$ and $\alpha_{n}=\omega_{\mathbf{i}}$ one has for all $p \geq 1$, all $\mathbf{i}=1, \ldots, \mathbf{i}^{*}$ and all integer $n \geq 3$

$$
\begin{array}{r}
\sup _{j=2,3} \mathbb{P}_{f}\left\{\sup _{D, Q \in \mathcal{Q}_{\delta_{n}}, D \neq Q}\left[\left|\xi_{h_{\mathbf{i}}, D}^{(j),(\mathbf{i})}(x)\right|-\frac{1}{3} \mathbf{C} L^{2} h_{\mathbf{i}}^{\beta}\right]>0\right\} \\
\leq \frac{2}{e^{8}}\left(\frac{\omega_{\mathbf{i}-1} \mathbf{n}_{\mathbf{i}}}{\mathbf{n}_{\mathbf{i}-1} \omega_{\mathbf{i}}}\right)^{\frac{p \beta}{2 \beta+1}} ; \\
\sup _{j=2,3} \mathbb{E}_{f}\left\{\sup _{D, Q \in \mathcal{Q}_{\delta_{n}}, D \neq Q}\left[\left|\xi_{h_{\mathbf{i}}, D}^{(j),(\mathbf{i})}(x)\right|-\frac{1}{3} \mathbf{C} L^{2} h_{\mathbf{i}}^{\beta}\right]_{+}\right\}^{p} \leq C^{\prime \prime} h_{\mathbf{i}}^{p \beta},
\end{array}
$$

imsart-generic ver. 2014/10/16 file: rotation-arXiv.tex date: February 26, 2020 
where $C^{\prime \prime}:=C^{\prime \prime}(p, \mathcal{K}, \beta, L, \alpha)>0$. Note finally that for any $\mathbf{i}=1, \ldots, \mathbf{i}^{*}$

$$
\omega_{\mathbf{i}} \leq L^{\frac{2}{2 \beta+1}}(9+3 \alpha)^{\frac{2 \beta+1 / 2}{2 \beta+1}} C(\beta) \mathbf{n}_{\mathbf{i}} \delta_{n} \sqrt{h_{\mathbf{i}}} .
$$

Thus, in view of (5.9) and (5.10) with $\epsilon=\epsilon_{3}=L^{-\frac{4}{2 \beta+1}}(9+4 \alpha)^{-\frac{2 \beta+2}{2 \beta+1}}[C(\beta)]^{-1}$ and $\alpha_{n}=\omega_{\mathbf{i}}$ one has for all $p \geq 1$, all $\mathbf{i}=1, \ldots, \mathbf{i}^{*}$ and all integer $n \geq 3$

$$
\begin{aligned}
& \mathbb{P}_{f}\left\{\sup _{D, Q \in \mathcal{Q}_{\delta_{n}}, D \neq Q}\left[\left|\xi_{h_{\mathbf{i}}, D}^{(1),(\mathbf{i})}(x)\right|-\frac{1}{3} \mathbf{C} L^{2} h_{\mathbf{i}}^{\beta}\right]>0\right\} \\
& \leq 6 e^{-8\left(\frac{\omega_{\mathbf{i}-1} \mathbf{n}_{\mathbf{i}}}{\mathbf{n}_{\mathbf{i}-1} \omega_{\mathbf{i}}}\right)^{\frac{p \beta}{2 \beta+1}} ;} \\
& \mathbb{E}_{f}\left\{\sup _{D, Q \in \mathcal{Q}_{\delta_{n}}, D \neq Q}\left[\left|\xi_{h_{\mathbf{i}}, D}^{(1),(\mathbf{i})}(x)\right|-\frac{1}{3} \mathbf{C} L^{2} h_{\mathbf{i}}^{\beta}\right]\right\}^{p} \leq C^{\prime \prime \prime} h_{\mathbf{i}}^{p \beta},
\end{aligned}
$$

where $C^{\prime \prime \prime}:=C^{\prime \prime \prime}(p, \mathcal{K}, \beta, L, \alpha)>0$. Proposition 3 is proved.

\section{References}

Amato, U., Antoniadis, A., Samarov, A. And Tsybakov, A. (2001) Noisy independent factor analysis model for density estimation and classification. Electronic Journal of Statistics, 4, 707-736.

Chen, H. (1991). Estimation of a projection-pursuit type regression model. Ann. Stat. 19, 142-157.

Goldenshluger, A. and Lepski, O.V. (2009). Structural adaptation via

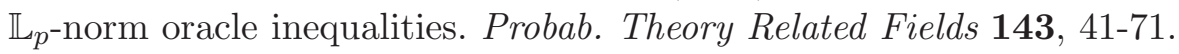

Goldenshluger, A. and Lepski, O.V. (2012). General selection rule from the family of linear estimators. Theory Probab. Appl., 57, 2, 257-277.

Golubev, G.K (1991) Asymptotically minimax estimation of a regression function in an additive model. Probl. Inform. Transm. 28, 101-112.

Horowitz, J. And Mammen, E. (2007) Rate-optimal estimation for a general class of non-parametric regression models with unknown link function. Ann. Stat. 35, 2589-2619.

Hristache, M., Juditsky, A., Polzehl, J., Spokoiny, V. (2001) Structure adaptive approach for dimension reduction. Ann. Stat. 29, 1537-1566.

Huber, P. (1985). Projection pursuit. With discussion. Ann. Stat. 13, 435525.

Juditsky, A.B., Lepski, O.V., Tsybakov, A.B. (2009) Nonparametric estimation of composite functions. Ann. Stat. 37, 3, 1360-1404. 
Kerkyacharian, G., Lepski, O. and Picard, D. (2007). Non linear estimation in anisotropic multiindex denoising. Sparse case. Probab. Theory Appl. 52, 1, 150-171.

LEPSKI, O.V. (2013). Multivariate density estimation under sup-norm loss: oracle approach, adaptation and independence structure. Ann. Statist. 41, 2, 1005-1034.

Lepski, O.V. And Serdyukova, N. (2014) Adaptive estimation under single-index constraint in a regression model. Ann. Stat. 42, 1, 1-28.

LePski, O.V. (2015). Adaptive estimation over anisotropic functional classes via oracle approach. Ann. Statist. 43, 3, 1178-1242.

Rebelles, G. (2015a). Pointwise adaptive estimation of a multivariate density under independence hypothesis. Bernoulli 21, 4, 1984-2023.

Rebelles, G. (2015b). $L_{p}$ adaptive estimation of an anisotropic density under independence hypothesis. Electronic Journal of Statistics 9, 106134.

Houdré, C. and Reynaud-Bouret, P. (2003). Exponential Inequalities, with Constants, for U-statistics of Order Two. Progress in Probability, 56, 55-69.

Samarov, A. and Tsybakov, A. (2004). Nonparametric independent component analysis. Bernoulli, 10, 4, 2004, 565-582.

Samarov, A. and Tsybakov, A. (2007). Aggregation of density estimators and dimension reduction. Advances in Statistical Modeling and Inference, 233-251, Ser. Biostat., 3, World Sci. Publ., Hackensack, NJ.

Stone, C.J. (1985). Additive regression and other nonparametric models. Ann. Stat. 13, 689-705. 